\title{
Ethnobotanical survey of cosmetic plants used in Marquesas Islands (French Polynesia)
}

Xénia Jost ${ }^{1}$, Jean-Luc Ansel ${ }^{2}$, Gaël Lecellier ${ }^{3,4}$, Phila Raharivelomanana ${ }^{5}$ and Jean-François Butaud ${ }^{6 *}$ (D)

\begin{abstract}
Background: Cosmetic plants and their uses have often been neglected in ethnobotanical surveys which focus mainly on plants with medicinal or food uses. Thus, this survey was carried out to specifically investigate cosmetics in a small community and to establish a cosmetopoeia, based on the model of pharmacopoeia for medicinal plants. The geographic spread of the survey covered the Marquesas Islands, one of the five archipelagos of French Polynesia (Pacific Ocean). This archipelago was also recently investigated for its pharmacopoeia.
\end{abstract}

Methods: This survey is based on individual interviews of Marquesan informants on the islands of Tahiti (Society archipelago) and Nuku Hiva (Marquesas archipelago). The methodological approach was semi-directive with open-ended questions based on cosmetic criteria (application area, cosmetic use, plant). Before each interview, researchers and the informant signed a Prior Informed Consent (PIC). Quantitative analyses were performed using basic statistics and the indice of Fidelity Level (FL).

Results: Twenty-eight informants from five of the six inhabited Marquesan islands were interviewed and yielded more than 500 cosmetic recipes. Marquesan cosmetopoeia included 79 plant taxa, of which 5\% are Marquesan endemics, 23\% are indigenous, $28 \%$ are Polynesian introductions and $44 \%$ are modern introductions. Among the introduced species, half were cultivated whereas the other half were weedy species. Most of the plants were abundant and only eight species were considered rare, of which four were Marquesan endemics. Main cosmetic plants were identified through informant citations and fidelity levels, and included Calophyllum inophyllum, Cananga odorata, Citrus aurantiifolia, Cocos nucifera, Curcuma longa, Gardenia taitensis, Mentha spp., Ocimum basilicum, Rauvolfia nukuhivensis and Santalum insulare var. marchionense. The most referred application areas were skin, hair and private parts whereas the main cosmetic uses were perfume, hydration, medicinal care and healing.

Conclusions: Through this survey, Marquesan cosmetopoeia was investigated in detail and uncovered a majority of introduced and abundant plants, and a minority of endemic and rare plants which required proper management to avoid future shortage. The well known perfumed coconut oil or monoi appeared as the main Marquesan cosmetic preparation either for the skin and the hair. Several plants and preparations warrant scientific investigations for their originality.

Keywords: Cosmetopoeia, Cosmetics, French Polynesia, Marquesas Islands, Pacific Ocean, Ethnobotanical survey

\footnotetext{
* Correspondence: jfbutaud@hotmail.com

${ }^{6}$ Consultant in forestry and Polynesian botany, B.P. 52832-98716 Pirae, Tahiti,

French Polynesia

Full list of author information is available at the end of the article
} 


\section{Background}

French Polynesia belongs to Eastern Polynesia and is located in the centre of the Pacific Ocean, between latitudes $7-28^{\circ} \mathrm{S}$ and longitudes $134-155^{\circ} \mathrm{W}$, with an exclusive economic zone of around 4.8 millions $\mathrm{km}^{2}$ and a total land area of $3521 \mathrm{~km}^{2}$ [1]. This French overseas country is composed of 118 islands and 5 archipelagos, namely the Society Islands (where the well known islands of Tahiti and Bora Bora are situated), the Austral Islands, the Tuamotu archipelago (only atolls), the Gambier Islands and the Marquesas Islands, for a population of 268,270 inhabitants in 2012 [2]. The Marquesas archipelago, subject of the present survey, is located in the North-East of French Polynesia (Fig. 1): it is the least populated (9264 inhabitants) and the most isolated from continents in the world. With its thirteen islands accounting for a total land area of $1050 \mathrm{~km}^{2}$, Marquesas Islands constitute $30 \%$ of all Polynesian territory, but only six islands are inhabited with $3.5 \%$ of Polynesian population. The Marquesas archipelago is usually divided into the Southern Marquesas Islands including Hiva Oa, Tahuata and Fatu Hiva (3504 inhabitants; $479 \mathrm{~km}^{2}$ ), and the Northern Marquesas Islands with Nuku Hiva, Ua Pou and Ua Huka (5760 inhabitants; $531 \mathrm{~km}^{2}$ ). Located between latitudes $7-10^{\circ} \mathrm{S}$, its climate is nearly dry tropical with average annual rainfalls of 1000-1800 $\mathrm{mm}$ and an average annual temperature of about $26{ }^{\circ} \mathrm{C}$ at sea level [3]. The Marquesan flora is well known with 350 native species including 187 endemic species (53\% Marquesan endemism), around 65 species

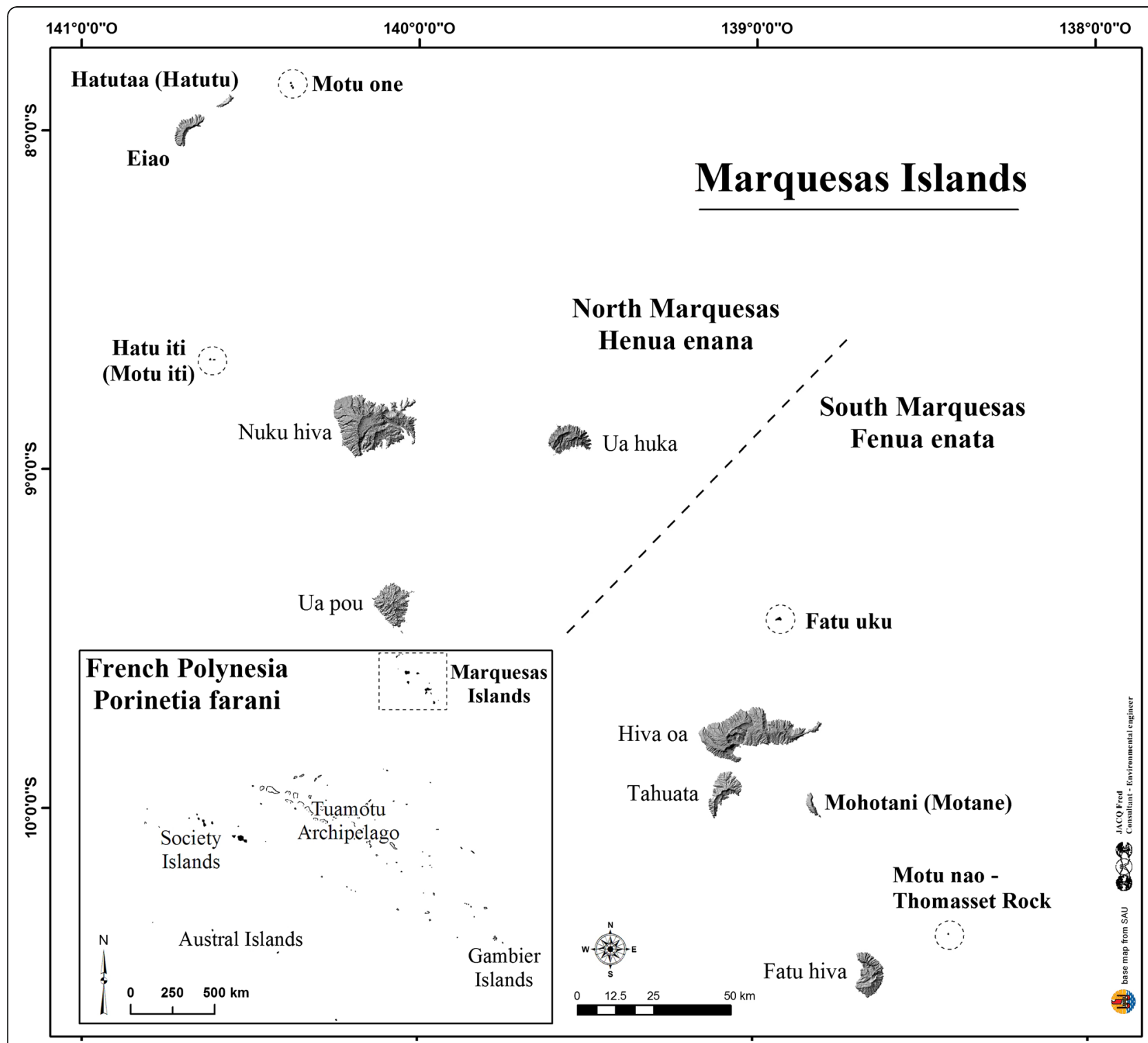

Fig. 1 Map of the Marquesas Islands in French Polynesia 
introduced during Polynesian migrations more than 1000 years ago (Polynesian introductions) and probably more than 800 species introduced more recently since the end of the 18th century and the European rediscovery of the archipelago (modern introductions) [4-7].

Cosmetopoeia consists of studying the traditional uses of raw materials, such as plants or minerals, for cosmetic purposes, analogous to a pharmacopoeia for medicinal plants $[8,9]$. It aims at gathering a better knowledge of this world cultural heritage, preserving those uses and promoting the biosourcing potential of natural ingredients for body care products. Traditional uses of cosmetic plants belong and are specific to each community from whom they originated, and particularly for the Polynesians inhabiting Pacific Ocean islands. Indeed, French Polynesia and particularly the Marquesas Islands [10], which possess a strong cultural identity [11], is well known throughout the world for perfumed coconut oil called monoi [12]. Natural fragrance is an important aspect of Marquesan beauty standards. Fragrant flowers are also omnipresent in traditional cosmetic preparations (mono' $i$ in Tahitian or pani in Marquesan) and can be directly applied to the hair or the skin.

The implementation of ethnobotanical studies dedicated to cosmetic plants is necessary due to their underrepresentation in such studies and the lack of details on their preparations and their uses [9, 10, 12-15]. Thus, the present survey reports the first field investigation of French Polynesian cosmetopoeia and focuses on traditional Marquesan plants used in cosmetics. It follows up an ethnobotanical investigation of Marquesan pharmacopoeia that was conducted previously by Girardi et al. [16].

\section{Methods}

\section{Geographic coverage}

Ethnobotanical surveys were performed on Tahiti (Society Islands) and Nuku Hiva (main island of the Marquesas archipelago) with Marquesan traditional practitioners from Nuku Hiva, Ua Pou and Ua Huka in the Northern
Marquesas, and Tahuata and Fatu Hiva in the Southern Marquesas (Table 1).

\section{Ethnobotanical data collection}

Informants were interviewed in Tahiti in May-July 2014 and June 2015, and in Nuku Hiva between 5-18 August 2014. In Tahiti, we interviewed them in their homes, as numerous Marquesans are living in Tahiti, and in an annual handicraft exhibition, gathering craftsmen from most of the Marquesas Islands come to the town of Pirae. In Nuku Hiva, we led our interviews in their homes as well, in all the villages of the island: Taiohae, the main village, Taipivai, Hooumi, Hatiheu and Aakapa. Regarding the investigations in Tahiti, we kept only coherent Marquesan preparations free of Tahitian influences (only Marquesan plant names, plants found in Marquesas Islands). All informants were selected and consulted for their rich knowledge of plants and traditional uses but also because they still continue to formulate cosmetic preparations for their friends, family or for tourists. We also focused on informants interviewed by Girardi et al. [16] for the Marquesan pharmacopoeia and on the informants recommended by members of "Académie marquisienne" or Marquesan Academy, specialists in Marquesan language and culture.

Before starting the interview, the interviewers had to introduce themselves and explain clearly to the informant the aims of the study. Prior to each interview process, a free informed consent form (PIC, Prior Informed Consent) had to be signed by both interviewer and informant (topic, objectives, goals of the study, respect of confidentiality); this PIC dealt also with access and benefit-sharing (ABS) agreements with the commitment taken by the researchers to associate individual informants, or a representative entity grouping them, to eventual assertion of Intellectual Property rights (patent) and commercial applications from cosmetic plant biochemical studies. The majority of interviews were individual, sometimes with two or three persons if translation was required when the informant spoke only

Table 1 Informants data and island of origin

\begin{tabular}{|c|c|c|c|c|c|}
\hline Marquesas & Island & Number of informants & Female proportion of informants & Inhabitants & Female proportion of inhabitants \\
\hline North & Nuku Hiva & 17 & $76 \%$ & 2966 & $47 \%$ \\
\hline North & Ua Pou & 2 & $50 \%$ & 2173 & $50 \%$ \\
\hline North & Ua Huka & 1 & $100 \%$ & 621 & $48 \%$ \\
\hline North & Total 3 islands & $20(71 \%)$ & $75 \%$ & $5760(62 \%)$ & $48 \%$ \\
\hline South & Hiva Oa & 0 & - & 2190 & $47 \%$ \\
\hline South & Tahuata & 2 & $100 \%$ & 703 & $49 \%$ \\
\hline South & Fatu Hiva & 6 & $100 \%$ & 611 & $47 \%$ \\
\hline South & Total 3 islands & $8(29 \%)$ & $100 \%$ & $3504(38 \%)$ & $47 \%$ \\
\hline Marquesas & Total 6 islands & 28 & $82 \%$ & 9264 & $48 \%$ \\
\hline
\end{tabular}


in Marquesan language. There were no refusals to participate, to sign the PIC form or to answer our questions.

The methodological approach was semi-directive with open-ended questions. The proposed method offered a list of allegations per target or per use. The survey was based on three standards: application area (hair, body, face) with practices specific to each area of application, uses (care, protection, hygiene, embellishment and perfume) with allegation lists linked to each application area, and ethnobotany. The interviewer chose an application area, and then asked open-ended questions per target (for example, if "body" as area, then skin, stomach, breasts, etc. as target). The interviews sought to determine the type of plants used, purposes of utilization, parts used, treated problems, modes of preparation and method of administration. An allegation is a use for a specific target, for example: anti-dandruff care for hair. If two different persons quote an identical allegation, we counted two allegation reports. Likewise, if two different preparations indicated an identical allegation, we counted two allegation reports. As the investigation progressed, all allegations proposed in the list were compiled and analysed. We took into account all the data given by informants, even including some allegations not present in the cosmetic list: these allegations were usually considered as "pharmacopoeia allegations". Therefore, one preparation can present several application areas, several targets, and/or several allegations.

\section{Plant identification}

The identification of each plant used for cosmetic treatments (quoted by its Marquesan name or showed to the interviewer) was facilitated by previous work done on Marquesan pharmacopoeia with numerous informants in common in both studies and with voucher specimens deposited at PAP herbarium in Tahiti [16], by botanical booklets aiming at linking Latin and Marquesan plant names $[5,6]$ and by the last author, botanist, who checked plant names and pictures reported by the interviewers. Several species of mints and frangipanis were clustered into unique taxa (ethnospecies), respectively Mentha spp. and Plumeria spp., because these plants are morphologically very close and are equally used for the same cosmetic uses in similar recipes.

\section{Data analysis}

The concept of an "ethnobotanical event" adapted from Tardio et al. [17] by Girardi et al. [16] for the study of Marquesan herbal medicine was implemented with the definition of allegation reports. A table gathering all data obtained from the investigations (informant, recipe, species, plant part, target, cosmetic use, allegation) was built to compile in different lines each allegation report so that each parameter could be analyzed independently. Another table was built with informant features: name, age, date and place of interview, number of recipes, occupation. Before initiating any analysis, grouping of allegations and various parts of plants were done in the first completed data table in order to homogenize all data; for example: treat injuries/heal wounds/tend scratches = healing, or grain/pod/fruit = seed. From this network, other tables and graphs were realised as well as statistical analysis with XLSTAT software (2014.4.02).

One of the most popular indexes in ethnopharmacology is the fidelity level (FL) $[18,19]$ and is implemented to quantify, for a group of people, the importance of one plant for a specific treatment. This index is calculated using the following formula FL $(\%)=100$ NIUE/NIU, where NIUE is the number of informants quoting one plant species for a particular use, and NIE is the number of informants quoting that plant for any use. The more the value of $\mathrm{FL}$ is close to 1 , the higher is the number of informants that used this plant species for that particular use. This index answers the question: "Which use is associated to this particular plant?"

Concerning the informants data, a correlation with a scatter plot in XLSTAT has been tested between parameters "number of recipes" and "number of allegations". A two-dimensional graph was built: the vertical axis represents the "number of recipes" value, and the horizontal axis represents the "number of allegations" value.

\section{Results and Discussion}

\section{Informants and geographical coverage}

A total of 28 Marquesan informants were interviewed, 23 women (82\%) and 5 men (18\%), ranging from 33 to 84 years old (Table 1). More women were interviewed than men, but it was not intentional: informants possessing a high knowledge about plants and their uses were targeted and were selected by Marquesan resource persons (Marquesan Academy or other informants). It is a fact that essentially women prepare and use cosmetic recipes, except for tattoo and that their traditional cosmetic knowledge was inherited from their mother or their grandmother, mostly from female lineage. Moreover, from the interviews, body care seems to be fundamentally a concern among women either for the well being of their children or themselves.

Relative to the geographical coverage, aiming to establish comparisons, we tried to balance interviews according to demographic distribution between Northern and Southern Marquesas, as these two sub-archipelagos are generally distinguished for language and cultural differences [20]. This intention was nearly achieved with $71 \%$ (56-86\% with a confidence interval of 95\%) of the informants coming from Northern Marquesas, which represents $62 \%$ of the whole archipelago population (Table 1). 
Overview on the knowledge of the 28 informants is given in Table 2 with their geographical origin, and their number of recipes, plant species used and allegation reports. For example, informant 29 came from the Northern Marquesas, gave 230 allegations in 24 different recipes using 28 distinct plant species. Thus, the higher the number of recipes/allegations is, the richer the informant knowledge is. This also provides an idea of the diversity of used plants in the 527 collected recipes.

On average, each informant gave 19 recipes (between 6 and 29), involving 20 plant species (between 5 and 30), and resulting in 115 allegation reports (between 9 and 230). No significant differences of knowledge between Northern and Southern Marquesas can be put in evidence, especially if Northern informants 12 and 13, who were males interviewed without the special recommendations of Marquesan Academy, are put aside. If the variations are not geographical, it appears that they are clearly linked with the gender, men having a quarter of recipes less, using $40 \%$ less plants and resulting in half the number of allegations in comparison with women (Table 2). This explains why the number of male informants is low: knowledge on cosmetic plants is fundamentally shared among Marquesan women today, even if some men (number 23 educated by its mother and number 31 the elder -84 years old), constituting the exceptions, have a rather similar knowledge.

\section{Taxonomy of cosmetic plants}

According to the interviews, 79 taxa (including species, subspecies and varieties) of cosmetic plants were documented for uses involving different plant parts; for convenience, the word species will be used instead of taxa or taxon in the text henceforth. Scientific name, botanical family name, biogeographical status, names given in Northern or Southern Marquesas, number of informants using them and other botanical data were recorded for each cosmetic plant species (Table 3). These species belong to 47 families, the most represented being Apocynaceae (4), Euphorbiaceae (4), Lamiaceae (4), Poaceae (4), Rutaceae (4), Convolvulaceae (3), Fabaceae (3), Malvaceae (3), Rubiaceae (3) and Zingiberaceae (3). Among the genera, Citrus (4), Gardenia (2), Ipomoea (2) and Zingiber (2) encompass several cosmetic species; the genera Fagraea, Mentha and Plumeria could also be mentioned as they comprised several very similar species or cultivars. It is interesting to observe that among the seven tropical tree family quoted by Ansel et al. [8] as being the more quoted in the literature for cosmetic uses in the world, four are also the main ones in the Marquesas, namely Fabaceae, Malvaceae, Rubiaceae and Rutaceae. At a lower level, genera Gardenia and Morinda (Rubiaceae), Citrus (Rutaceae), Hibiscus (Malvaceae), Cocos (Arecaceae), Calophyllum (Calophyllaceae) and
Artocarpus and Ficus (Moraceae) appear in both studies. This exhibits the congruence of cosmetopoeia of the Marquesas Islands and at least the Asia-Pacific region.

\section{Biogeographical status of cosmetic plants}

The flora of Marquesas Islands is usually classified according to the biogeographical status of each plant, between native species which were not introduced by human, Polynesian introductions which were introduced during Polynesian migrations more than one thousand years ago, and modern introductions which were introduced since the end of the 18th century and the arrival of European vessels. Moreover, native species can be divided into endemic species, which are restricted to the Marquesas Islands, and indigenous species, which are also found outside this archipelago. Thus, the majority of cosmetic plants corresponded to modern introductions ( $44 \%, 35$ species) whereas $28 \%$ of them ( 22 species) were constituted by Polynesian introductions, 23\% (18 species) were indigenous, and only $5 \%$ (4 species) were endemic to Marquesas archipelago (Fig. 2). This fact showed the importance of introduced species in the present cosmetopoeia and more precisely the value of Polynesian introductions (breadfruit tree, red hibiscus, Tahitian gardenia, cordyline, Malay apple, candlenut tree, shampoo ginger, etc.), which were purposely introduced for their uses and the rapid integration of modern introductions, proof of continuous innovation. The number of native species, accounting for around a quarter of all the cosmetic species, was comparatively low, reflecting perhaps the relative poverty of Marquesan flora compared with other Pacific archipelagos floras [21]. Nevertheless, four endemic plants were parts of these native species and showed the importance of restricted species; however it is worth noting that three of these endemics have close relatives in most of the Pacific in the same species (Alyxia stellata) or in the same genus (Euphorbia, Santalum), indicating the possibility of knowledge transfer through archipelagos.

\section{Naturalization status of cosmetic plants}

Regarding the naturalization status of the 57 introduced or alien species (Fig. 3), 22 species (39\%) are always cultivated, 9 species (16\%) are subspontaneous or spreading not far away from former cultivation place, 2 species (4\%) are adventive or weeds and 24 species (41\%) are naturalized and spreading without any human help. Thus, more than $60 \%$ of the introduced species were not cultivated and grew quite freely in disturbed areas like gardens and roads or among natural vegetation. Interestingly, cultivation is not reserved for introduced species which are not naturalized: indeed, two native species (Cordia subcordata and Premna serratifolia), nine subspontaneous species and seven naturalized species are 
Table 2 Geographical origin (in bracket, number of informants), number of recipes, number of plants and number of allegations per informant (M: male, others: female)

\begin{tabular}{|c|c|c|c|c|}
\hline Marquesas & Informant number & Number of recipes & Number of plants & Number of allegations \\
\hline \multirow[t]{20}{*}{ North } & 5 & 17 & 21 & 94 \\
\hline & $12(\mathrm{M})$ & 6 & 5 & 9 \\
\hline & $13(\mathrm{M})$ & 9 & 9 & 42 \\
\hline & 16 & 22 & 24 & 117 \\
\hline & 17 & 10 & 16 & 97 \\
\hline & 18 & 15 & 21 & 75 \\
\hline & 19 & 17 & 16 & 84 \\
\hline & 20 & 15 & 16 & 73 \\
\hline & 22 & 16 & 24 & 129 \\
\hline & $23(\mathrm{M})$ & 26 & 25 & 77 \\
\hline & 24 & 17 & 30 & 156 \\
\hline & 25 & 10 & 8 & 63 \\
\hline & 26 & 25 & 21 & 190 \\
\hline & $27(\mathrm{M})$ & 15 & 13 & 59 \\
\hline & 28 & 19 & 16 & 81 \\
\hline & 29 & 24 & 28 & 230 \\
\hline & 30 & 29 & 24 & 204 \\
\hline & 31 (M) & 20 & 13 & 111 \\
\hline & 32 & 28 & 27 & 171 \\
\hline & 33 & 20 & 25 & 118 \\
\hline \multirow[t]{3}{*}{ North (20) } & Maximum & 29 & 30 & 230 \\
\hline & Average & 18.0 & 19.1 & 109.0 \\
\hline & Minimum & 6 & 5 & 9 \\
\hline \multirow[t]{8}{*}{ South } & 3 & 25 & 24 & 59 \\
\hline & 6 & 17 & 25 & 198 \\
\hline & 7 & 16 & 20 & 112 \\
\hline & 9 & 17 & 19 & 87 \\
\hline & 10 & 26 & 21 & 127 \\
\hline & 21 & 18 & 24 & 172 \\
\hline & 35 & 23 & 24 & 171 \\
\hline & 36 & 25 & 22 & 124 \\
\hline \multirow[t]{3}{*}{ South (8) } & Maximum & 26 & 25 & 198 \\
\hline & Average & 20.9 & 22.4 & 131.3 \\
\hline & Minimum & 16 & 19 & 59 \\
\hline \multirow[t]{3}{*}{ Marquesas (28) } & Maximum & 29 & 30 & 230 \\
\hline & Average & 18.8 & 20.0 & 115.4 \\
\hline & Minimum & 6 & 5 & 9 \\
\hline \multirow[t]{3}{*}{ Female (23) } & Maximum & 29 & 30 & 230 \\
\hline & Average & 19.6 & 21.6 & 127.5 \\
\hline & Minimum & 10 & 8 & 59 \\
\hline \multirow[t]{3}{*}{ Male (5) } & Maximum & 26 & 25 & 111 \\
\hline & Average & 15.2 & 13.0 & 59.6 \\
\hline & Minimum & 6 & 5 & 9 \\
\hline
\end{tabular}


Table 3 Cosmetic plants cited by informants with botanical information

\begin{tabular}{|c|c|c|c|c|c|c|c|c|c|c|}
\hline Family & Scientific name & $\begin{array}{l}\mathrm{N} / \\
\mathrm{S} \\
(1)\end{array}$ & $\begin{array}{l}\text { Biogeographical } \\
\text { status (2) }\end{array}$ & Cultivation & $\begin{array}{l}\text { Abundance } \\
\text { (3) }\end{array}$ & $\begin{array}{l}\text { Naturalization } \\
\text { status (4) }\end{array}$ & $\begin{array}{l}\text { Type of } \\
\text { plant }\end{array}$ & $\begin{array}{l}\text { Local name }(\mathrm{N}) \\
\text { (1) }\end{array}$ & $\begin{array}{l}\text { Local name } \\
\text { (S) (1) }\end{array}$ & $\begin{array}{l}\text { Number of } \\
\text { informants }\end{array}$ \\
\hline Amaranthaceae & Achyranthes aspera $\mathrm{L}$. var. aspera & $\mathrm{N}$ & Pol & No & 3 & Nat & Shrublet & mokio & - & 2 \\
\hline Asteraceae & Ageratum conyzoides L. & $\begin{array}{l}\mathrm{N} / \\
\mathrm{S}\end{array}$ & Mod & No & 1 & Nat & Herbaceous & $\begin{array}{l}\text { mei'e, mei'e } \\
\text { rore }\end{array}$ & mei'e, putara & 6 \\
\hline Euphorbiaceae & Aleurites moluccanus (L.) Willd. & $\begin{array}{l}\mathrm{N} / \\
\mathrm{S}\end{array}$ & Pol & No & 3 & Nat & Tree & 'ama & 'ama, ti'a'iri & 5 \\
\hline Xanthorrhoeaceae & Aloe vera (L.) Burm.f. & $\begin{array}{l}\mathrm{N} / \\
\mathrm{S}\end{array}$ & Mod & Yes & 2 & Cult & Herbaceous & - & - & 1 \\
\hline Apocynaceae & $\begin{array}{l}\text { Alyxia stellata var. marquesensis (F.Br.) Fosberg \& } \\
\text { Sachet }\end{array}$ & N & End & No & 5 & - & Shrub & mei'e papa & - & 5 \\
\hline Amaranthaceae & Amaranthus viridis $\mathrm{L}$. & S & Pol & No & 1 & Nat & Herbaceous & - & upo'oti'i & 2 \\
\hline Bromeliaceae & Ananas comosus (L.) Merr. & $\begin{array}{l}\mathrm{N} / \\
\mathrm{S}\end{array}$ & Mod & Yes & 1 & Subsp & Herbaceous & - & - & 3 \\
\hline Apiaceae & Anethum graveolens $\mathrm{L}$. & $\begin{array}{l}\mathrm{N} / \\
\mathrm{S}\end{array}$ & Mod & Yes & 2 & Cult & Herbaceous & taretare & - & 1 \\
\hline Marratiaceae & Angiopteris evecta (J.G. Forst.) Hoffm. & $\begin{array}{l}\mathrm{N} / \\
\mathrm{S}\end{array}$ & Ind & No & 3 & - & Fern & pa'ahei, puhei & pa'ahei & 2 \\
\hline Annonaceae & Annona muricata $\mathrm{L}$. & $\begin{array}{l}\mathrm{N} / \\
\mathrm{S}\end{array}$ & Mod & Yes & 1 & Subsp & Tree & - & - & 4 \\
\hline Moraceae & Artocarpus altilis (Parkinson ex Z) Fosberg & $\begin{array}{l}\mathrm{N} / \\
\mathrm{S}\end{array}$ & Pol & Yes & 1 & Subsp & Tree & ‘uru, maiore & 'uru & 3 \\
\hline Plantaginaceae & Bacopa monnieri (L.) Wettst. & N & Ind & No & 3 & - & Herbaceous & heiotona & - & 1 \\
\hline Lecythidaceae & Barringtonia asiatica (L.) Kurz & N & Ind & No & 3 & - & Tree & hutu & - & 1 \\
\hline Bixaceae & Bixa orellana $\mathrm{L}$. & $\begin{array}{l}\mathrm{N} / \\
\mathrm{S}\end{array}$ & Mod & Yes & 3 & Subsp & Shrub & roku, peni & roku, peni & 1 \\
\hline Calophyllaceae & Calophyllum inophyllum L. & $\begin{array}{l}\mathrm{N} / \\
\mathrm{S}\end{array}$ & Pol & No & 2 & Nat & Tree & temanu & $\begin{array}{l}\text { tamanu, } \\
\text { temanu }\end{array}$ & 10 \\
\hline Annonaceae & Cananga odorata (Lam.) Hook.f. \& Thomson & $\begin{array}{l}\mathrm{N} / \\
\mathrm{S}\end{array}$ & Mod & Yes & 1 & Nat & Tree & moto'i & moto'i & 4 \\
\hline Sapindaceae & Cardiospermum halicacabum $\mathrm{L}$. & N & Pol & No & 3 & Adv & Vine & komoka & - & 2 \\
\hline Caricaceae & Carica papaya $\mathrm{L}$. & $\begin{array}{l}\mathrm{N} / \\
\mathrm{S}\end{array}$ & Mod & Yes & 1 & Nat & Shrub & - & - & 1 \\
\hline Poaceae & Centotheca lappacea (L.) Desv. & S & Pol & No & 3 & Nat & Herbaceous & - & 'ohe'ohe & 1 \\
\hline Apocynaceae & Cerbera manghas L. & N & Ind & No & 3 & - & Tree & 'eva & - & 1 \\
\hline Poaceae & Chrysopogon zizanioides (L.) Roberty & $\begin{array}{l}\mathrm{N} / \\
\mathrm{S}\end{array}$ & Mod & Yes & 2 & Cult & Herbaceous & metie, mouku & mouku & 8 \\
\hline Rutaceae & Citrus aurantiffolia (Christm. \& Panz.) Swingle & $\begin{array}{l}\mathrm{N} / \\
\mathrm{S}\end{array}$ & Mod & Yes & 1 & Subsp & Shrub & hitoro & - & 10 \\
\hline
\end{tabular}


Table 3 Cosmetic plants cited by informants with botanical information (Continued)

\begin{tabular}{|c|c|c|c|c|c|c|c|c|c|c|}
\hline Rutaceae & Citrus hystrix DC. & $\begin{array}{l}\mathrm{N} / \\
\mathrm{S}\end{array}$ & Mod & Yes & 3 & Cult & Shrub & - & remene & 5 \\
\hline Rutaceae & Citrus maxima (Burm.) Merr. & N & Mod & Yes & 2 & Cult & Shrub & - & - & 2 \\
\hline Rutaceae & Citrus $x$ sinensis (L.) Osbeck & $\begin{array}{l}\mathrm{N} / \\
\mathrm{S}\end{array}$ & Mod & Yes & 3 & Cult & Tree & anani & - & 2 \\
\hline Arecaceae & Cocos nucifera $\mathrm{L}$. & $\begin{array}{l}\mathrm{N} / \\
\mathrm{S}\end{array}$ & Pol & Yes & 1 & Nat & Tree & 'ehi & e'ehi & 12 \\
\hline Rhamnaceae & Colubrina asiatica (L.) Brongn. var. asiatica & $\begin{array}{l}\mathrm{N} / \\
\mathrm{S}\end{array}$ & Ind & No & 3 & - & Vine & tutu & tutu & 4 \\
\hline Commelinaceae & Commelina diffusa Burm.f. & N & Mod & No & 1 & Nat & Herbaceous & heiotona & - & 1 \\
\hline Cordiaceae & Cordia subcordata Lam. & $\begin{array}{l}\mathrm{N} / \\
\mathrm{S}\end{array}$ & Ind & Yes & 2 & - & Tree & tou & tou & 1 \\
\hline Asparagaceae & Cordyline fruticosa (L.) A. Chev. & $\begin{array}{l}\mathrm{N} / \\
\mathrm{S}\end{array}$ & Pol & Yes & 1 & Nat & Shrub & 'auti & 'auti & 2 \\
\hline Cucurbitaceae & Cucumis anguria $\mathrm{L}$. & N & Mod & No & 4 & Nat & Vine & kokopa kira & - & 1 \\
\hline Zingiberaceae & Curcuma longa $\mathrm{L}$. & $\begin{array}{l}\mathrm{N} / \\
\mathrm{S}\end{array}$ & Pol & Yes & 2 & Subsp & Herbaceous & 'eka & 'ena, re'a & 6 \\
\hline Cyperaceae & Cyperus mindorensis (Steud.) Huygh & $N$ & Pol & No & 1 & Nat & Herbaceous & $\begin{array}{l}\text { punie poko } \\
\text { tava'i'ie, mutie }\end{array}$ & - & 2 \\
\hline Fabaceae & Erythrina variegata $\mathrm{L}$. & N & Pol & No & 3 & Nat & Tree & kenae & - & 2 \\
\hline Euphorbiaceae & Euphorbia hirta L. & N & Mod & No & 1 & Nat & Herbaceous & $\begin{array}{l}\text { heeheeamata, } \\
\text { pokea, } \\
\text { eaeamata }\end{array}$ & - & 7 \\
\hline Euphorbiaceae & Euphorbia sachetiana (J. Florence) Govaerts & $\begin{array}{l}\mathrm{N} / \\
\mathrm{S}\end{array}$ & End & No & 5 & - & Shrublet & - & - & 1 \\
\hline Gentianaceae & $\begin{array}{l}\text { Fagraea berteroana A. Gray ex Benth. (syn. F. } \\
\text { berteroana var. marquisensis Fosberg \& Sachet) } \\
\text { native form }\end{array}$ & $\begin{array}{l}\mathrm{N} / \\
\mathrm{S}\end{array}$ & Ind & No & 4 & - & Tree & pua 'enana & $\begin{array}{l}\text { pua, pua } \\
\text { ho'ovai } \\
\text { 'enata }\end{array}$ & 2 \\
\hline Gentianaceae & $\begin{array}{l}\text { Fagraea berteroana A. Gray ex Benth. (syn. F. } \\
\text { longituba M.L. Grant) introduced form }\end{array}$ & $S$ & Mod & Yes & 2 & Cult & Tree & - & $\begin{array}{l}\text { pua ho'ovai } \\
\text { vaikeka'a }\end{array}$ & 1 \\
\hline Moraceae & Ficus prolixa G. Forst. var. prolixa & $\begin{array}{l}\mathrm{N} / \\
\mathrm{S}\end{array}$ & Ind & No & 3 & - & Tree & aoa & aoa & 2 \\
\hline Rubiaceae & Gardenia jasminoides Ellis & N & Mod & Yes & 2 & Cult & Shrub & taina & - & 2 \\
\hline Rubiaceae & Gardenia taitensis DC. & $\begin{array}{l}\mathrm{N} / \\
\mathrm{S}\end{array}$ & Pol & Yes & 1 & Cult & Shrub & Tia'e, tiare & tiare & 10 \\
\hline Malvaceae & Gossypium hirsutum var. taitense (Parl.) Roberty & N & Ind & No & 4 & - & Shrub & haha'avai & - & 2 \\
\hline Malvaceae & Hibiscus rosa-sinensis $\mathrm{L}$. & $\mathrm{S}$ & Pol & Yes & 1 & Subsp & Shrub & - & 'oute pupu & 3 \\
\hline Convolvulaceae & Ipomoea batatas (L.) Lam. & $\begin{array}{l}\mathrm{N} / \\
\mathrm{S}\end{array}$ & Pol & Yes & 1 & Cult & Vine & - & 'uma'a & 2 \\
\hline
\end{tabular}


Table 3 Cosmetic plants cited by informants with botanical information (Continued)

\begin{tabular}{|c|c|c|c|c|c|c|c|c|c|c|}
\hline Convolvulaceae & Ipomoea pes-caprae subsp. brasiliensis (L.) Ooststr. & N & Ind & No & 3 & - & Vine & pohue tatahi & - & 1 \\
\hline Oleaceae & Jasminum grandiflorum L. & $\begin{array}{l}\mathrm{N} / \\
\mathrm{S}\end{array}$ & Mod & Yes & 1 & Cult & Vine & pitate & pitate & 6 \\
\hline Fabaceae & Leucaena leucocephala (Lam.) de Wit & $\mathrm{N}$ & Mod & No & 1 & Nat & Shrub & - & - & 1 \\
\hline Euphorbiaceae & Manihot esculenta Crantz & N & Mod & Yes & 1 & Cult & Shrublet & ara & - & 6 \\
\hline Lamiaceae & Mentha spp. & $\begin{array}{l}\mathrm{N} / \\
\mathrm{S}\end{array}$ & Mod & Yes & 1 & Cult & Herbaceous & mati & mati & 18 \\
\hline Polypodiaceae & Microsorum grossum (Langsd. \& Fisch.) S.B. Andrews & $\begin{array}{l}\mathrm{N} / \\
\mathrm{S}\end{array}$ & Ind & No & 2 & - & Fern & $\begin{array}{l}\text { papamoko, } \\
\text { metuapua'a }\end{array}$ & papamo'o & 7 \\
\hline Rubiaceae & Morinda citrifolia L. & $\begin{array}{l}\mathrm{N} / \\
\mathrm{S}\end{array}$ & Ind & No & 1 & - & Shrub & noni & noni, nono & 7 \\
\hline Fabaceae & Mucuna sloanei Fawc. \& Rendle var. sloanei & N & Ind & No & 3 & - & Vine & 'auto'u & papanuiaohe & 3 \\
\hline Musaceae & Musa $\times$ paradisiaca $\mathrm{L}$. & S & Pol & Yes & 1 & Cult & Herbaceous & - & - & 1 \\
\hline Lamiaceae & Ocimum basilicum L. & $\begin{array}{l}\mathrm{N} / \\
\mathrm{S}\end{array}$ & Mod & Yes & 1 & Nat & Shrublet & $\begin{array}{l}\text { miri, mini, miri } \\
\text { keka'a }\end{array}$ & miri & 8 \\
\hline Convolvulaceae & Operculina brownii Ooststr. & N & Ind & No & 4 & - & Vine & piatakiohoau & - & 1 \\
\hline Cactaceae & Opuntia cochenillifera (L.) Mill. & N & Mod & Yes & 2 & Cult & Shrub & - & - & 1 \\
\hline Pandanaceae & Pandanus tectorius Parkinson ex $Z$ var. tectorius & $\begin{array}{l}\mathrm{N} / \\
\mathrm{S}\end{array}$ & Ind & No & 3 & - & Tree & hinako & hinano & 5 \\
\hline Passifloraceae & Passiflora foetida $\mathrm{L}$. & S & Mod & No & 2 & Nat & Vine & - & pu'u moina & 2 \\
\hline Lauraceae & Persea americana Mill. & N & Mod & Yes & 1 & Subsp & Tree & - & - & 1 \\
\hline Phyllanthaceae & Phyllanthus amarus Schumach. \& Thonn. & $\begin{array}{l}\text { N/ } \\
S\end{array}$ & Mod & No & 1 & Nat & Herbaceous & moemoe & 'au'iki, tuitui & 2 \\
\hline Solanaceae & Physalis angulata $\mathrm{L}$. & S & Pol & No & 2 & Adv & Herbaceous & - & kariri & 2 \\
\hline Lamiaceae & Plectranthus scutellarioides (L.) R.Br. & $\begin{array}{l}\text { N/ } \\
\text { S }\end{array}$ & Mod & Yes & 1 & Cult & Shrublet & terevete & terevete & 1 \\
\hline Apocynaceae & Plumeria spp. & $\begin{array}{l}\mathrm{N} / \\
\mathrm{S}\end{array}$ & Mod & Yes & 1 & Cult & Shrub & tipanie & tipanie & 1 \\
\hline Araliaceae & Polyscias scutellaria (Burm.f.) Fosberg & S & Mod & Yes & 1 & Cult & Shrub & - & kafeie & 1 \\
\hline Lamiaceae & Premna serratifolia L. & $\begin{array}{l}\mathrm{N} / \\
\mathrm{S}\end{array}$ & Ind & Yes & 1 & - & Shrub & va'ova'o & $\begin{array}{l}\text { 'avaro, } \\
\text { va'ova'o }\end{array}$ & 6 \\
\hline Myrtaceae & Psidium guajava $\mathrm{L}$. & $\begin{array}{l}\mathrm{N} / \\
\mathrm{S}\end{array}$ & Mod & No & 2 & Nat & Shrub & tuava & - & 2 \\
\hline Apocynaceae & $\begin{array}{l}\text { Rauvolfia nukuhivensis (Fosberg \& Sachet) Lorence \& } \\
\text { Butaud }\end{array}$ & N & End & No & 5 & - & Tree & tu'eiao & - & 11 \\
\hline Brassicaceae & $\begin{array}{l}\text { Rorippa sarmentosa (Sol. ex G. Forst. ex DC.) J.F. } \\
\text { Macbr. }\end{array}$ & $\begin{array}{l}\mathrm{N} / \\
\mathrm{S}\end{array}$ & Pol & No & 1 & Nat & Herbaceous & mahimahi & mahimahi & 1 \\
\hline Poaceae & Saccharum officinarum L. & & Pol & Yes & 2 & Cult & Herbaceous & - & - & 4 \\
\hline
\end{tabular}


Table 3 Cosmetic plants cited by informants with botanical information (Continued)

\begin{tabular}{|c|c|c|c|c|c|c|c|c|c|c|}
\hline & & $\begin{array}{l}N / \\
S\end{array}$ & & & & & & & & \\
\hline Santalaceae & $\begin{array}{l}\text { Santalum insulare var. marchionense (Skottsb.) } \\
\text { Skottsb. }\end{array}$ & $\begin{array}{l}\mathrm{N} / \\
\mathrm{S}\end{array}$ & End & No & 4 & - & Tree & puahi, ahi & puahi & 7 \\
\hline Sapindaceae & Sapindus saponaria $\mathrm{L}$. & $\begin{array}{l}\mathrm{N} / \\
\mathrm{S}\end{array}$ & Ind & No & 2 & - & Tree & koku'u & koku'u & 3 \\
\hline Asteraceae & Sigesbeckia orientalis L. & $\begin{array}{l}N / \\
S\end{array}$ & Pol & Yes & 2 & Nat & Herbaceous & niou & niou, riou & 2 \\
\hline Anacardiaceae & Spondias dulcis Sol. ex Parkinson & $\mathrm{N}$ & Mod & Yes & 2 & Cult & Tree & vi farani & - & 1 \\
\hline Myrtaceae & Syzygium malaccense (L.) Merr. \& L.M. Perry & $\begin{array}{l}N / \\
S\end{array}$ & Pol & Yes & 2 & Nat & Tree & 'ahi'a, kehika & $\begin{array}{l}\text { Kehi'a, kehi'a } \\
\text { 'enata, kehika }\end{array}$ & 2 \\
\hline Malvaceae & Thespesia populnea (L.) Sol. ex Corrêa & $\begin{array}{l}\text { N/ } \\
S\end{array}$ & Ind & No & 2 & - & Tree & miro, mi'o & mi'o & 4 \\
\hline Orchidaceae & Vanilla $\times$ tahitensis J.W. Moore & S & Mod & Yes & 2 & Subsp & Vine & - & - & 1 \\
\hline Poaceae & Zea mays $\mathrm{L}$. & N & Mod & Yes & 3 & Cult & Herbaceous & - & - & 1 \\
\hline Zingiberaceae & Zingiber officinale Roscoe & S & Mod & Yes & 2 & Cult & Herbaceous & re'a blanc & & 1 \\
\hline Zingiberaceae & Zingiber zerumbet (L.) Sm. & $\begin{array}{l}\mathrm{N} / \\
\mathrm{S}\end{array}$ & Pol & No & 3 & Nat & Herbaceous & kopuhi, 'opuhi & 'opuhi & 5 \\
\hline
\end{tabular}

(1) $\mathrm{N}=$ used in Northern Marquesas; $\mathrm{S}=$ used in Southern Marquesas

(2) Pol = Polynesian introduction; Mod = modern introduction; Ind = indigenous species; End = endemic to the Marquesas Islands

(3) 1 = very common; $2=$ common; $3=$ uncommon; $4=$ rare; 5 =very rare

(4) Nat = naturalized species; Adv = adventive, weedy species; Subsp = subspontaneous species; Cult = cultivated specie 


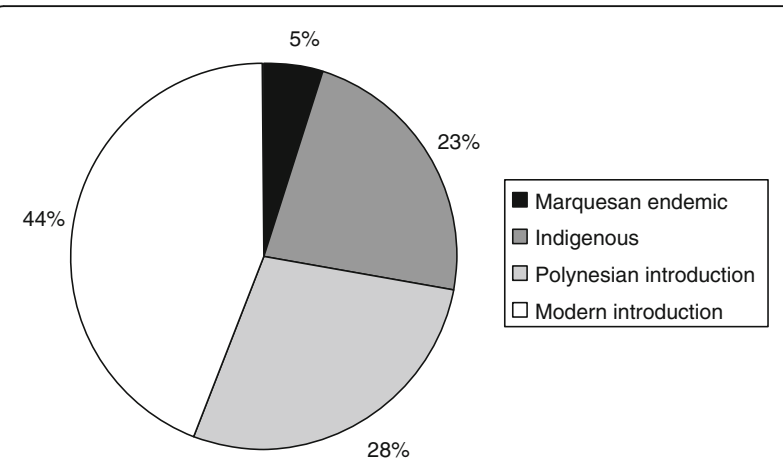

Fig. 2 Biogeographical status of cosmetic plants

also cultivated (Table 3); thus, in total, 40 species or half the cosmetic flora, can be obtained by cultivation which enables an easy access to species growing far form the villages or producing not enough materials in the wild. For example, the native shrub Premna serratifolia is scattered in dry to mesic forests and is not common enough to allow good harvest of inflorescences; as for the basil Ocimum basilicum, it is quite rare at the wild state. The case of the native tree Fagraea berteroana, growing in mid to high elevation wet forest, replaced in cosmetic recipes by an introduced cultivar, formerly known under the name $F$. longituba, which is easily cultivated at low elevation in drier places, is symptomatic of the optimization of the cosmetic ingredients harvest (here, the similar flowers of both species).

\section{Abundance and distribution}

Several criteria allowed to characterize plant "abundance" trait: accessibility (mainly distance from houses), and scarcity at the scale of island, valley or island. Precisions on the abundance categories used in Table 3 are given in Table 4. Thus, a cosmetic plant, which is easy to find close to houses, is given the value 1 , whereas the one, which is very difficult to find at the scale of the island, is given the highest value 5 .

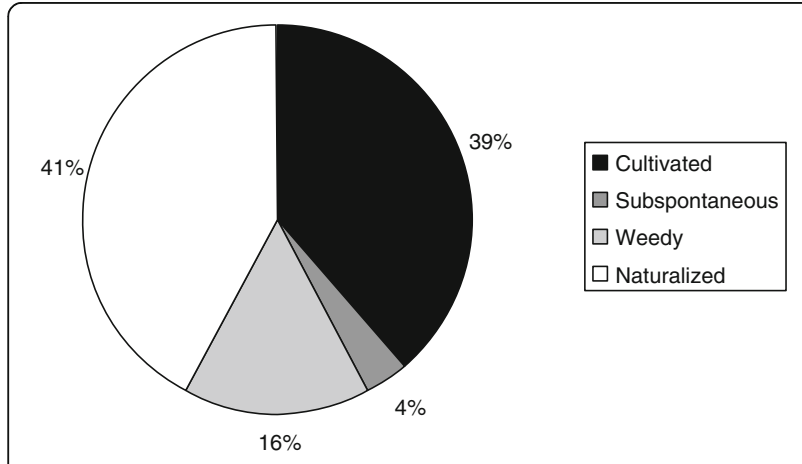

Fig. 3 Naturalization status of cosmetic plants
Two-thirds of Marquesan cosmetic plants are common to very common (Fig. 4), preventing any problem of overexploitation and associated shortage due to their use. Only eight species (10\%) are considered rare or very rare: they encompass the four Marquesan endemics plus three indigenous species (Fagraea berteroana, Operculina brownii, Gossypium hirsutum var. taitense) and one modern introduction (Cucumis anguria) restricted to dry areas. These seven rare native species are threatened mainly by exotic invasive species which alter their habitats (rats eating their fruits; wild horse, cattle and goats eating their barks, leaves or sprouts; invasive plants choking mother-plants and their regeneration) but some of them are also victims of overexploitation like sandalwood for its fragrant heartwood, or Rauvolfia nukuhivensis and Alyxia stellata var. marquesensis for their barks [22-24].

From a total of 79 plant species (Table 3), 24 species (30\%) are exclusively mentioned by people from Northern Marquesas (Rauvolfia nukuhivensis, Euphorbia hirta, Manihot esculenta, Alyxia stellata, etc.) and 10 species (13\%) by people from Southern Marquesas (Hibiscus rosa-sinensis, Physalis angulata, Passiflora foetida, Amaranthus viridis, etc.). Thus, 45 plant species are used for cosmetics in the whole archipelago whereas people from the North seem to possess a wider range of cosmetic species (69 species versus 55 in the South). It is noteworthy to point out that all these exclusive species grow in all the islands of the Marquesas and are mentioned only by one or two informants, except Rauvolfia nukuhivensis, which is well known by most of Nuku Hiva community. This species, nearly restricted to Nuku Hiva island in Northern Marquesas (only three living trees known on Ua Huka and probably extinct on Hiva $\mathrm{Oa})$, is frequently used in cosmetics, particularly for intimate hygiene of young girls. The difference between North and South Marquesas could be attributed for the most part to a faint cultural differentiation, as for the Marquesan language [20], but also for a small part to the longer time spent with the Northern Marquesan informants.

\section{Life forms of cosmetic plants}

Among the 79 cosmetic plants, the main life forms (Fig. 5) are trees (coconut tree, screwpine, banyan, sandalwood, Pacific rosewood, etc.) and herbaceous (turmeric, mint, pineapple, sugar cane, etc.) with 22 species for both (28\% each), followed by shrubs (Tahitian lime, Tahitian gardenia, frangipani, Polynesian cotton, etc.) with 18 species (23\%). Vines (vanilla, sweet potato, jasmine, etc.) are less common with 10 species (13\%). Shrublets (basil, tapioca, etc.) and ferns (Microsorum grossum, Angiopteris evecta) are the least used, with respectively $5(6 \%)$ and two (3\%) species. The fern flora, 
Table 4 Definitions of abundance categories

\begin{tabular}{lll}
\hline Value & Category & Definition \\
\hline 1 & Very common & $\begin{array}{l}\text { Easy to find close to all houses } \\
\text { Close to houses, but } \\
\text { not always all houses } \\
\text { Close to few houses, } \\
\text { but very scarce in the } \\
\text { natural environment }\end{array}$ \\
4 & Rare & $\begin{array}{l}\text { Hard to find in valleys } \\
\text { (inhabited area), easier } \\
\text { at the scale of the island } \\
\text { Hard to find at the scale } \\
\text { of the valley and the island }\end{array}$ \\
\hline
\end{tabular}

counting for around one third of the native Marquesan species, is then very much the minority among Marquesan cosmetopoeia.

\section{Plant parts used}

Plants can be used as a whole, especially for small species like Phyllanthus amarus or Cyperus mindorensis, or for specific parts. An aggregation of plant parts citations was created in order to conduct proper analyses on a few main categories (Fig. 6); for example, the "seed" category includes fruit, nut, kernel, infructescence or pod and the "flower" category includes inflorescence and flower bud. Coconut, which is almost omnipresent, is separated in its own category although it is a nut or seed. Among the different parts of cosmetic plants used by Marquesans, the most reported were the coconut (857 citations-25\%), flower (779-23\%) and leaf \& bud (693-21\%). It is remarkable to note the plant parts diversity with all the plant organs used including the wood for the sandalwood, the bark for Rauvolfia nukuhivensis or the stem for sugarcane. Moreover, for some plants, several parts are used for cosmetic recipes, like basil (Ocimum basilicum), from which flowers, leaves and seeds are used independently.

\section{Main cosmetic plants}

Six plant species are quoted by more than one third of the informants (Table 3): Mentha spp. (mints) 64\%, Cocos nucifera (coconut tree) 43\%, Rauvolfia nukuhivensis 39\%, Citrus aurantiifolia (Tahitian lime) 36\%, Gardenia taitensis (Tahitian gardenia or tiare) $36 \%$ and Calophyllum inophyllum (Alexandrian laurel or tamanu) $36 \%$.

Regarding the number of citations, Cocos nucifera is by far the main species with 805 citations (Fig. 7). This plant is used in many recipes as an excipient; which is an active principle vehicle for a lot of preparations containing several ingredients. Especially in cosmetopoeia, some excipients have cosmetic properties, and could therefore be also considered as active principles. For example, coconut water as well as coconut oil are the main excipients used to prepare cosmetic recipes, but can also be used for their own cosmetic properties. With 229 citations, Ocimum basilicum (basil) is the second most important cosmetic plant. The other species with more than 100 citations are Curcuma longa (turmeric-219 allegations), Santalum insulare var. marchionense (Marquesan sandalwood-215 allegations), Gardenia taitensis (162 allegations), Cananga odorata (ylang-ylang-133 allegations) and Citrus aurantiifolia (108 allegations).

These ten species constitute the bulk of the Marquesan cosmetopoeia, which is then composed of two endemic plants, four Polynesian introductions and four modern introductions.

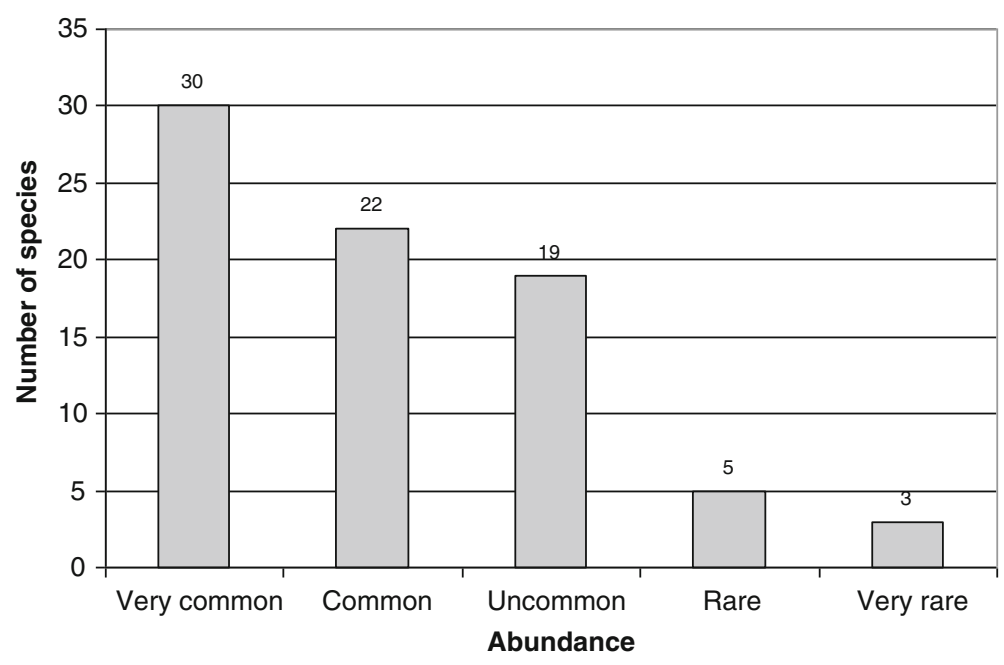

Fig. 4 Abundance of cosmetic plants 


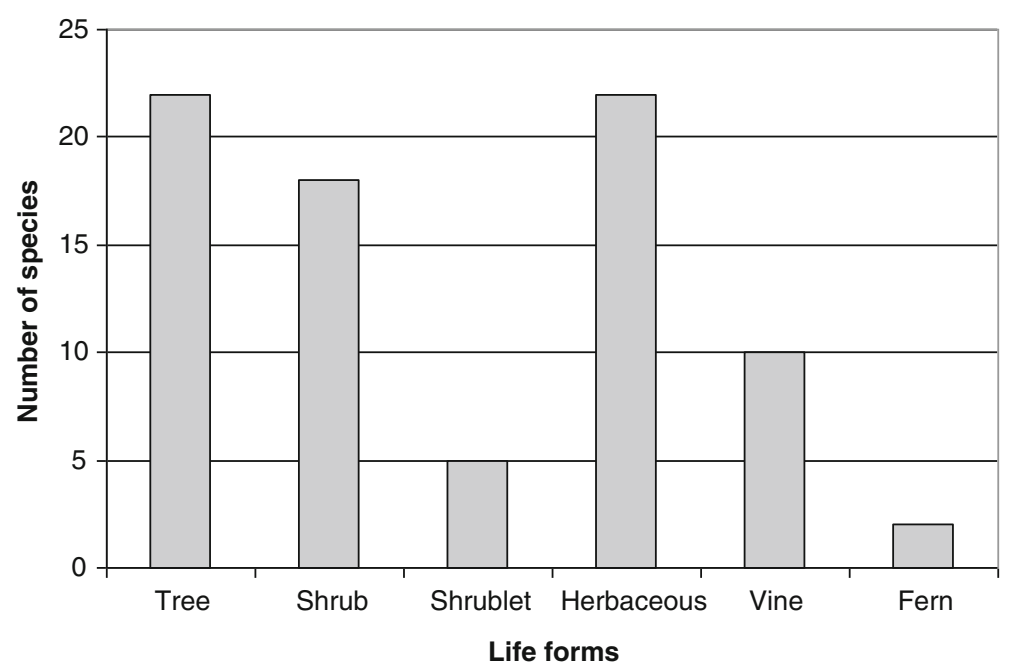

Fig. 5 Life forms of cosmetic plants

\section{Cosmetic recipes, allegations and uses}

The 28 interviews yielded a list of 527 recipes. This study focused on cosmetic uses and a majority of cosmetic allegations were observed. Nevertheless, among these recipes, $6 \%$ referred to pharmacopoeia, confirming that there are no clear boundaries in Marquesan culture between concepts of body/aesthetic care and medicinal care, as has been shown by Girardi et al. [16] studying Marquesan pharmacopoeia. Regarding posology, cosmetic recipes could be used every day unlike medicinal recipes. However, recipes addressing skin problems are applied for three days, similar to how a medicine may be applied. Dosages and quantities are not well defined or well measured: plant quantities are measured with handful, hand size or finger size. Adults and children were not subjected to the same dosages to avoid any risk of

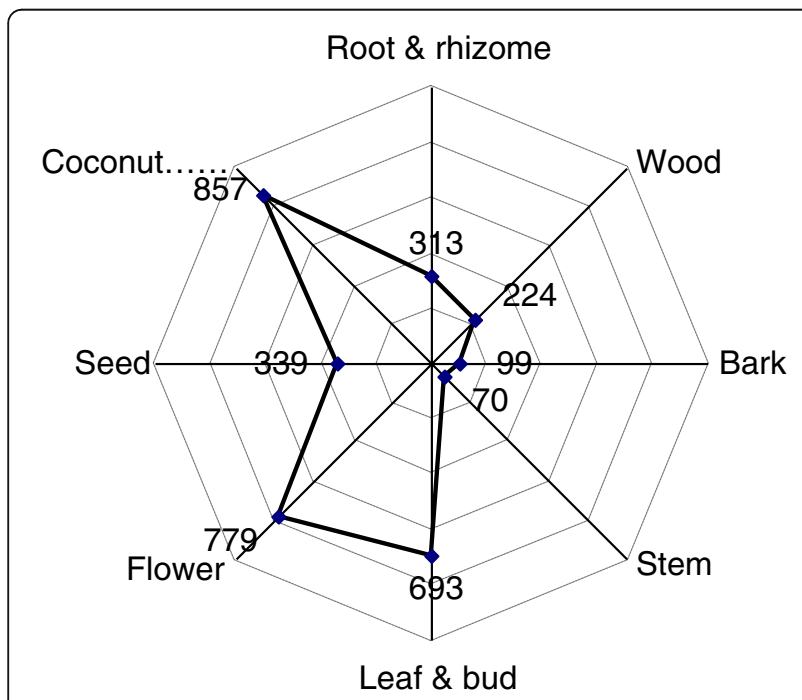

Fig. 6 Number of citations per plant parts category poisoning as the cosmetic preparations were age and/or weight dose-dependent. As for medicinal recipes [16], each cosmetic recipe contained several plant species (regularly five or more) and can be used on several application areas and for different uses. This leads to a logarithmical correlation between the number of recipes and the number of allegations per informant as shown on the scatter plot of Fig. 8, allegations number increasing exponentially with recipes number. Moreover, each informant knew around 20 cosmetic recipes, which account for around 100 allegations.

The most referred application areas (Fig. 9) are skin with 1630 allegations (48\% of all allegations) and hair with 986 allegations (29\%). Private parts come in third position with 163 allegations (5\%), followed by whole face $(4 \%)$, armpit (3\%) and lips \& mouth (3\%) whereas the other 15 application areas account each for less than $2 \%$ of all allegations. This analysis indicates key Polynesian cultural traits with the importance of skin and hair care but also a Marquesan originality with private parts as one of the main application areas [12-15].

The main cosmetic uses can be distinguished from minor ones in Fig. 10 where 37 cosmetic uses have been recognized. Most informants used plants, in cosmetics, for perfume (28\% of all allegations), hydration (23\%) and healing (5\%). Medicinal care was also quoted in $7 \%$ of allegations, indicating the close links between Marquesan cosmetopoeia and pharmacopoeia; among these, most visible body care actions could be related to aesthetic and by extension cosmetic concepts. Apart from these four main cosmetic uses, which account for $63 \%$ of all allegations, all the other cosmetic uses account for under $3 \%$ each of allegations. Marquesan cosmetopoeia appears to be based on the smell (perfume, deodorant, intimate hygiene, soap), on skin care (hydration, medicinal care, 


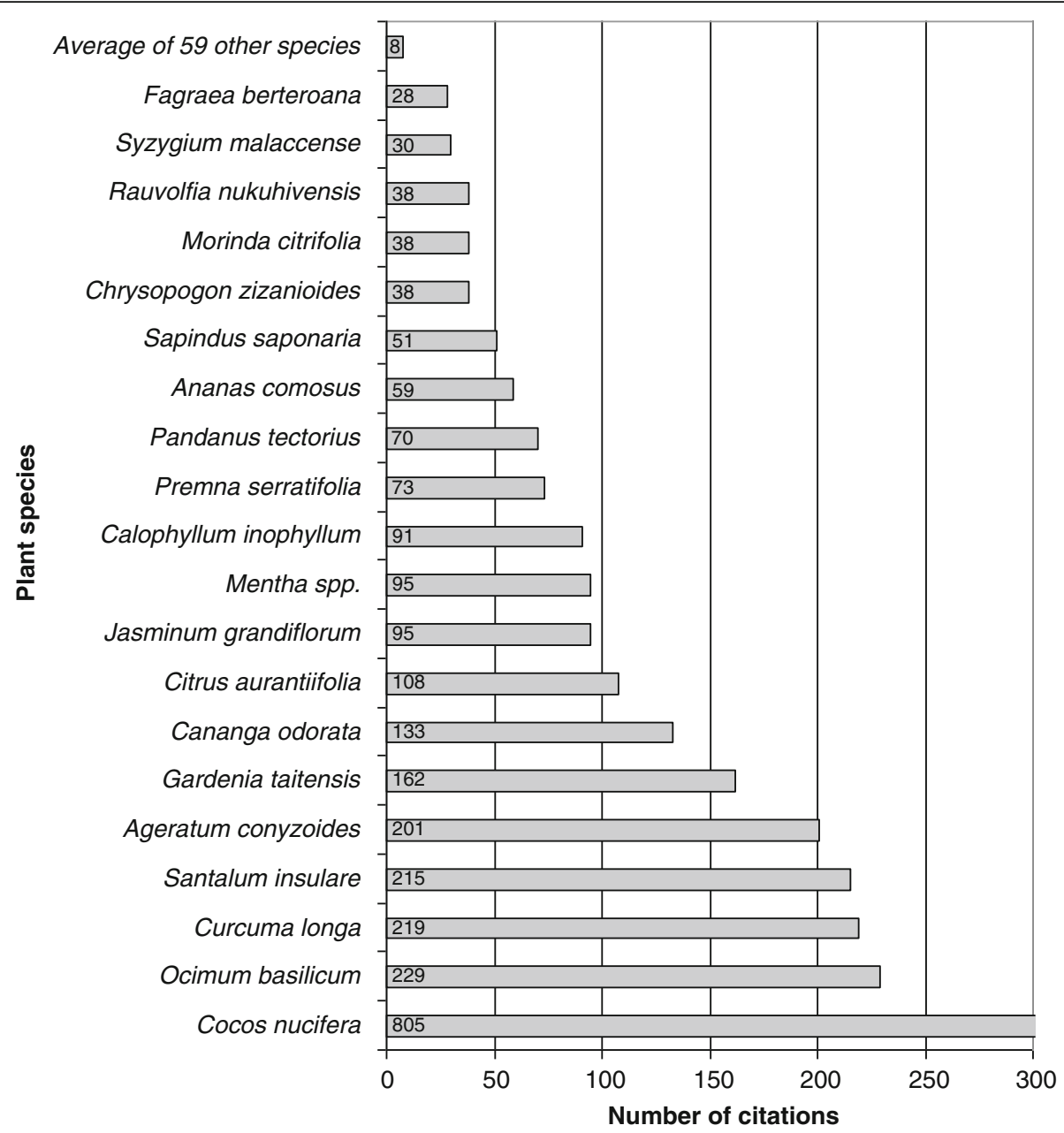

Fig. 7 Number of citations per plant species

healing, pimple care, mosquito repellent, etc.) and on hair care (anti-dandruff, hair conditioner, beneficial for hair growth, hair smoothing, shampoo). A summary of these detailed cosmetic uses in main categories for informants from Northern and Southern Marquesas confirms these characteristics (Fig. 11); moreover, it appears that cosmetic practices from both sub-archipelagos do not differ in terms of cosmetic use categories, both of them indicating care (52\%), perfume (27\%) and hygiene (10\%) as the main categories. Among cosmetic plants, 69

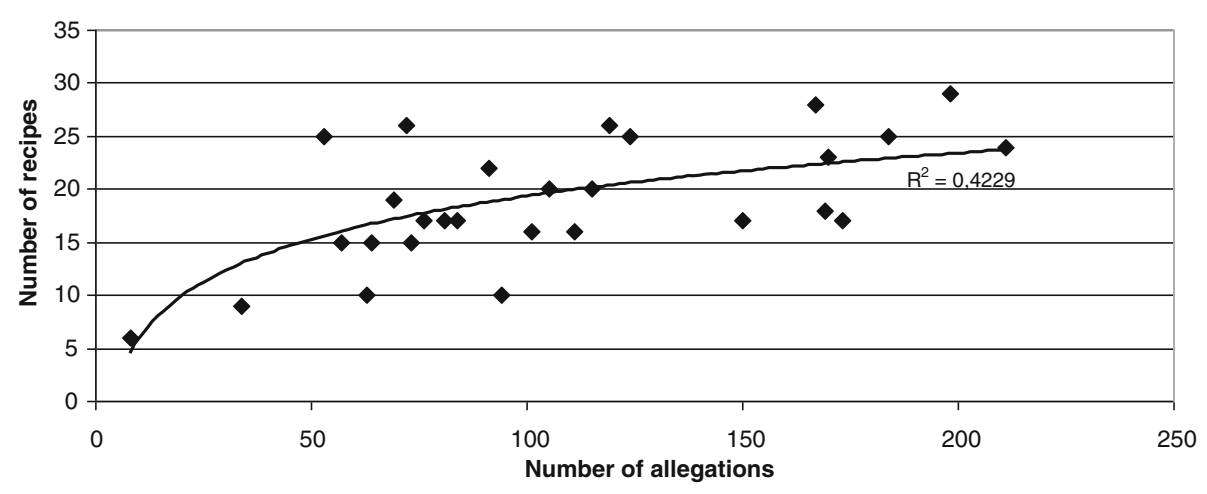

Fig. 8 Correlation between number of recipes and number of allegations 


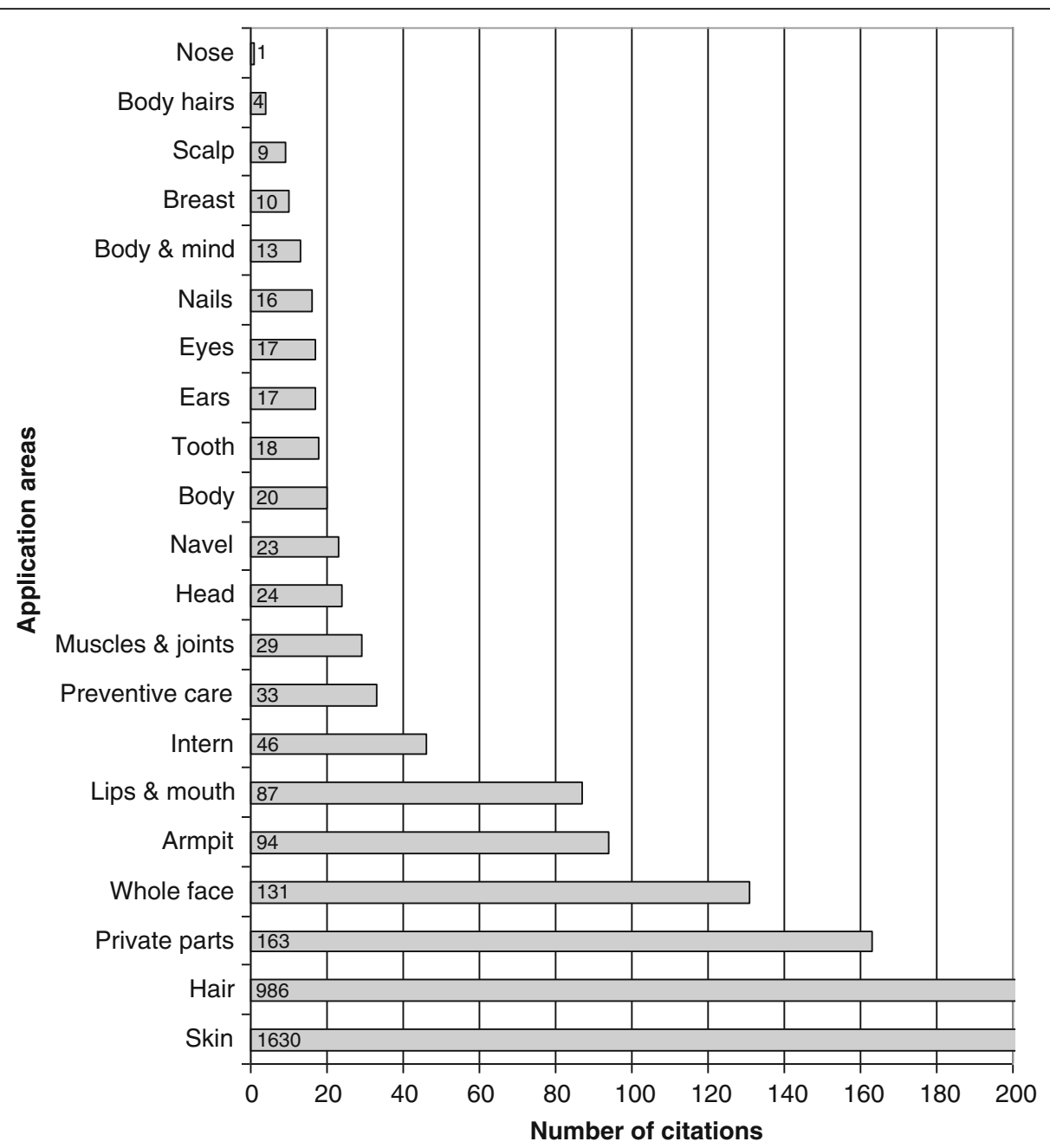

Fig. 9 Number of citations per application area

species $(87 \%)$ are involved in care category whereas 45 species (57\%) are used for hygiene and 43 (54\%) for perfume (Fig. 12); this indicates utilization of most cosmetic plants in several cosmetic use categories.

\section{Fidelity level of a species for a particular use}

Fidelity levels were calculated for the 575 pairs of cosmetic use - plant species in order to quantify the importance of a species for a particular use. Table 5 indicated the 31 fidelity levels higher than $65 \%$ related to use species associations known by more than one third of the informants. Thus 16 species and seven cosmetic uses appeared to be particularly linked. Fidelity levels were significantly high (several 100\% of FL) for several species (Ageratum conyzoides, Cananga odorata, Curcuma longa, Gardenia taitensis, Jasminum grandiflorum, Mentha spp., Ocimum basilicum, Pandanus tectorius and Santalum insulare) used in association with coconut oil for both perfume and hydration, the latter being probably mainly linked with Cocos nucifera oil. Two species were closely correlated with female intimate hygiene: the young nut of Cocos nucifera and the bark of Rauvolfia nukuhivensis, which were also put in evidence in Marquesan pharmacopoeia in preventive care [16]. Healing applications were used mostly with Calophyllum inophyllum seed oil, Cocos nucifera nut oil or Santalum insulare heartwood powder. Finally, the fruits of Citrus aurantifolia and the leaves of Syzygium malaccense are commonly used as deodorant and anti-cold sore and canker sore agents, respectively. Among these main fidelity levels, it is striking to note the presence of two Marquesan endemic plants (Rauvolfia nukuhivensis and Santalum insulare var. marchionense) among the six of Marquesan cosmetopoeia; this constitutes real originality and could lead to pertinent phytochemical studies as recently highlighted [25-27]. Regarding coconut oil, its omnipresence is related to its use as an excipient for fragrant preparations but also to its own virtues (active principle) in hydration for example. 


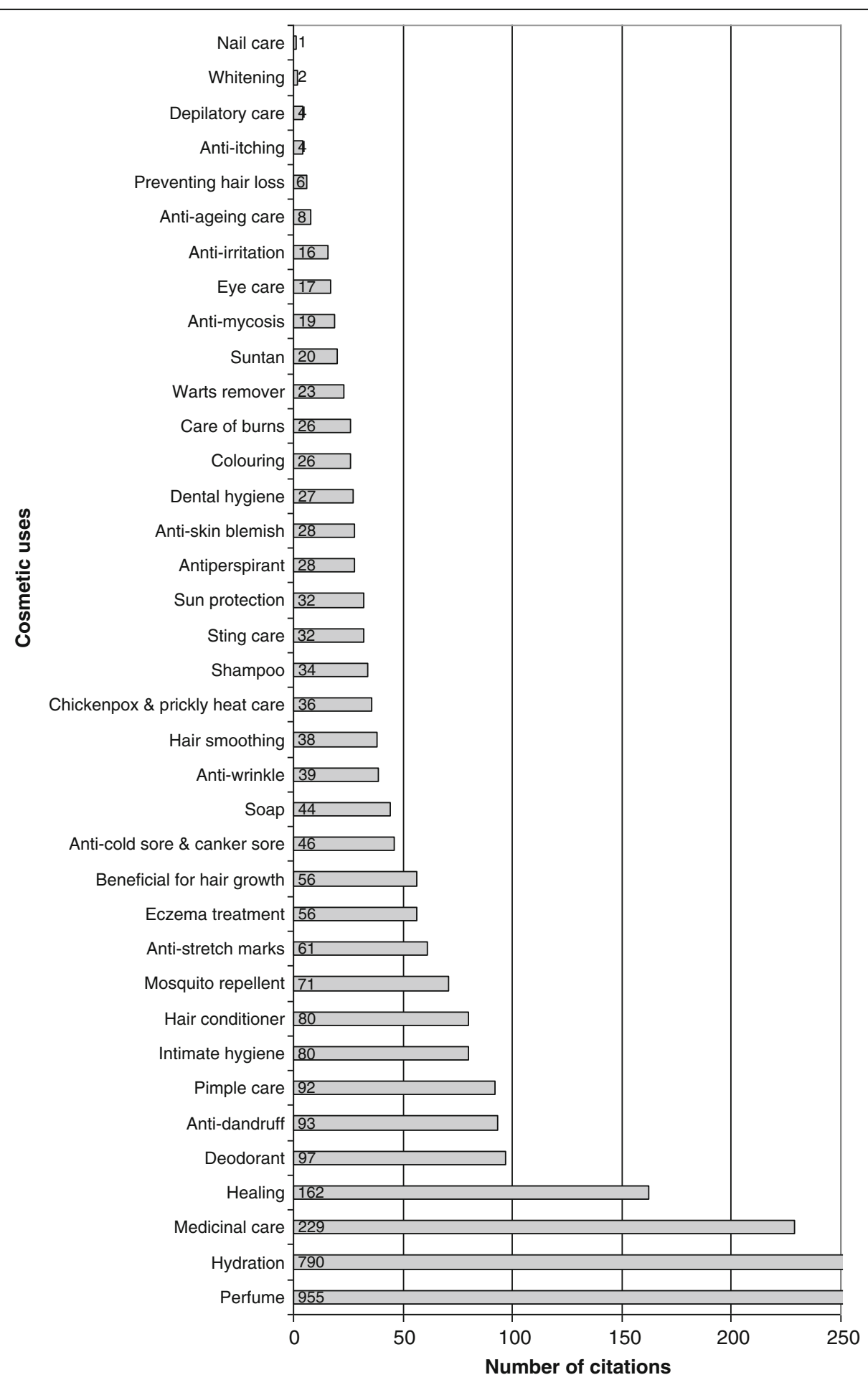

Fig. 10 Number of citations per cosmetic use

\section{Description of typical Marquesan cosmetic recipes}

Based on traditional Marquesan cosmetic concepts, care is provided through three main recipes categories: monoi, "paku" and "hoho".

\section{Monoi}

Monoi or mono'i in Tahitian, which means perfumed coconut oil, is also called pani in Marquesan and is known worldwide as a cosmetic oil. Among the three 


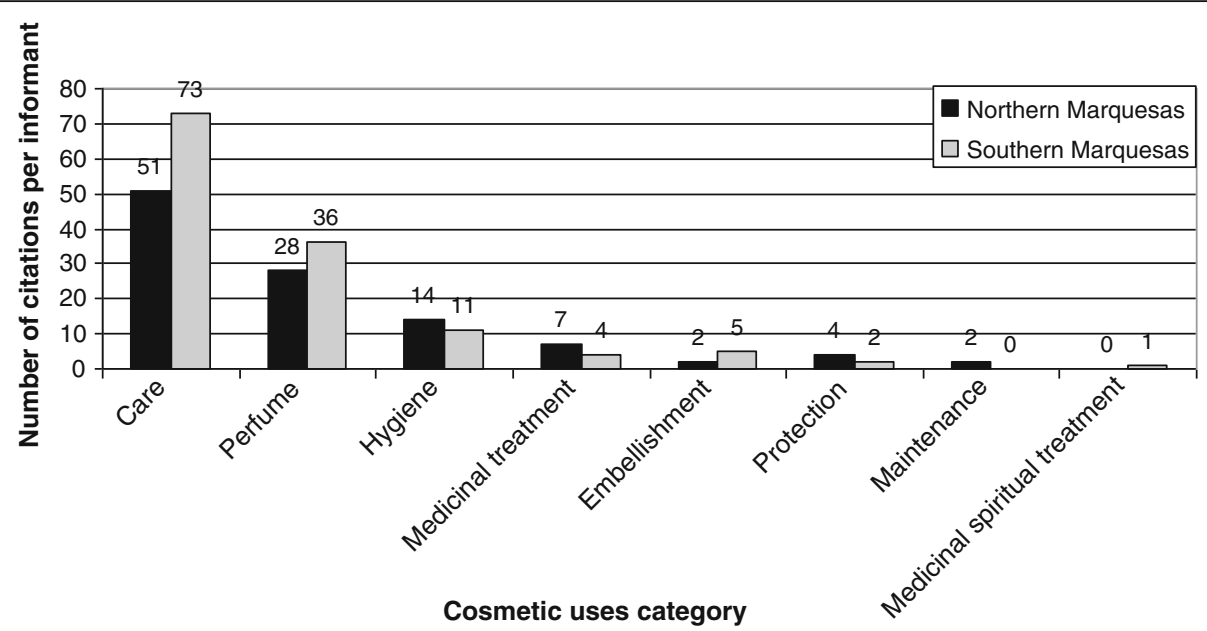

Fig. 11 Number of citations per informant for each category of cosmetic uses

categories, monoi takes the longest time to prepare: the preparation process can take from several days up to a week. Grated coconut, usually from a germinated coconut, is necessarily exposed to the sun in order to obtain the oil, which is later perfumed by the maceration of fresh plant materials for several days. The oil can also be recovered by sun exposure of the coconut milk coming from grated coconut pressing. Macerated plant materials are replaced every two or three days and are used either for their fragrance, like Tahitian gardenia (Gardenia taitensis), Spanish jasmine (Jasminum grandiflorum), Sweet basil (Ocimum basilicum) or for their therapeutic virtues like Alexandrian laurel or tamanu in Tahitian/temanu in Marquesan (Calophyllum inophyllum) or Polynesian sandalwood (Santalum insulare). Monoi can be made with only one plant (pani pitate, pani tia'e, pani temanu...) or with a combination of several species (pani kumuhei). Monoi is used for the softness and the care of body and hair, with fragrance as a key part. These preparations can be applied daily.

\section{Paku}

The term "paku" is mainly known in Northern Marquesas but informants from the whole archipelago have recipes that match its description. Two types of $p a k u$ are identified: the first is dedicated to the care and beauty of hair and skin; the second is mostly dedicated to children, even newborns, as a preventive treatment, in order to care cradle cap, to avoid bad smells and to limit vaginal discharges. It has also both medicinal and spiritual uses, the latter being to drive away evil spirits, particularly for babies. Paku preparations involve only fresh plants (one or several species), which are often crushed in coconut milk or grated coconut; usually, little (one to two hours) to no sun exposure is needed. This preparation is to be applied immediately by massage of the obtained juice on the targeted part of the body (head, armpit, private parts, all the body) and can be kept on the body

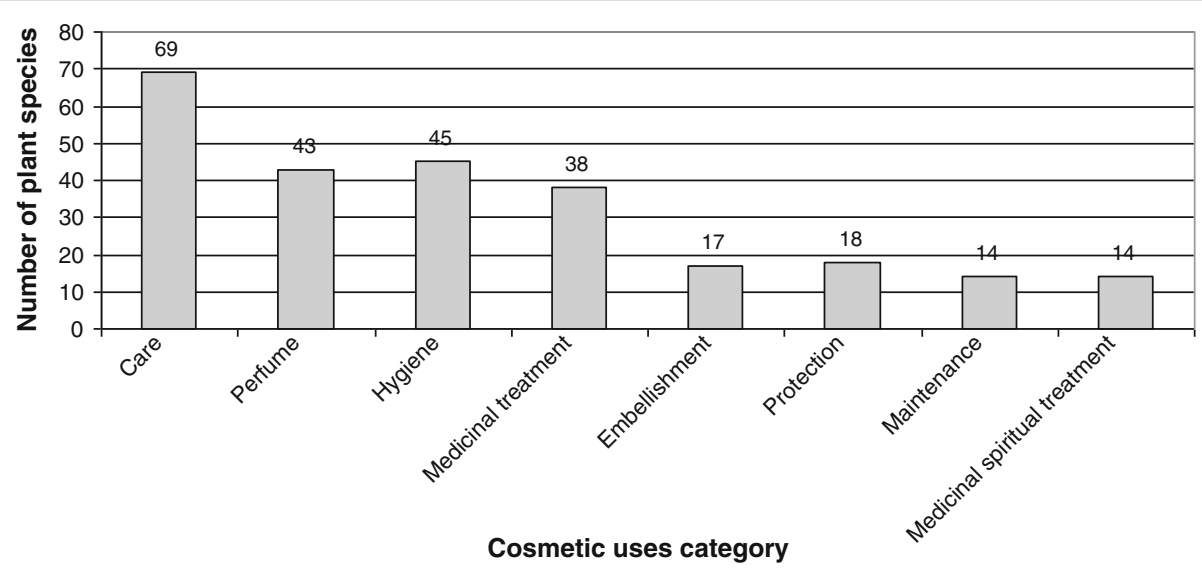

Fig. 12 Number of plant species per category of cosmetic uses 
Table 5 Main fidelity levels for Marquesan cosmetopoeia

\begin{tabular}{|c|c|c|c|c|}
\hline Cosmetic use & Plant species & NIUE & NIE & Fidelity level (\%) \\
\hline Hydration & Ageratum conyzoides & 17 & 20 & 85,0 \\
\hline Perfume & Ageratum conyzoides & 19 & 20 & 95,0 \\
\hline Perfume & Ananas comosus & 11 & 11 & 100,0 \\
\hline Healing & Calophyllum inophyllum & 13 & 20 & 65,0 \\
\hline Hydration & Cananga odorota & 18 & 20 & 90,0 \\
\hline Perfume & Cananga odorota & 20 & 20 & 100,0 \\
\hline Deodorant & Citrus aurantiifolia & 21 & 23 & 91,3 \\
\hline Healing & Cocos nucifera & 23 & 28 & 82,1 \\
\hline Hydration & Cocos nucifera & 27 & 28 & 96,4 \\
\hline Intimate hygiene & Cocos nucifera & 19 & 28 & 67,9 \\
\hline Perfume & Cocos nucifera & 27 & 28 & 96,4 \\
\hline Medicinal care & Cocos nucifera & 20 & 28 & 71,4 \\
\hline Hydration & Curcuma longa & 21 & 25 & 84,0 \\
\hline Perfume & Curcuma longa & 17 & 25 & 68,0 \\
\hline Hydration & Gardenia taitensis & 20 & 25 & 80,0 \\
\hline Perfume & Gardenia taitensis & 19 & 25 & 76,0 \\
\hline Hydration & Jasminum grandiflorum & 10 & 15 & 66,7 \\
\hline Perfume & Jasminum grandiflorum & 14 & 15 & 93,3 \\
\hline Hydration & Mentha spp. & 13 & 16 & 81,3 \\
\hline Perfume & Mentha spp. & 16 & 16 & 100,0 \\
\hline Hydration & Ocimum basilicum & 18 & 21 & 85,7 \\
\hline Perfume & Ocimum basilicum & 19 & 21 & 90,5 \\
\hline Hydration & Pandanus tectorius var. tectorius & 12 & 13 & 92,3 \\
\hline Perfume & Pandanus tectorius var. tectorius & 12 & 13 & 92,3 \\
\hline Perfume & Premna serratifolia & 12 & 16 & 75,0 \\
\hline Intimate hygiene & Rauvolfia nukuhivensis & 10 & 11 & 90,9 \\
\hline Perfume & Rauvolfia nukuhivensis & 10 & 11 & 90,9 \\
\hline Healing & Santalum insulare var. marchionense & 18 & 27 & 66,7 \\
\hline Hydration & Santalum insulare var. marchionense & 25 & 27 & 92,6 \\
\hline Perfume & Santalum insulare var. marchionense & 19 & 27 & 70,4 \\
\hline Anti-cold sore \& canker sore & Syzygium malaccense & 11 & 16 & 68,8 \\
\hline
\end{tabular}

for a whole day. Paku is not used daily but can be applied until the issue at hand is alleviated. The main plants used for paku are Ageratum conyzoides, Cocos nucifera, Colubrina asiatica var. asiatica, Curcuma longa, Gossypium hirsutum var. taitense and Sapindus saponaria; it should be noted that Colubrina asiatica and Sapindus saponaria are well known for their uses as soap.

\section{Hoho}

The term "hoho" is known only in the island of Fatu Hiva in Southern Marquesas. Only one type of hoho exists even if different plants can be used. Hoho is mainly aesthetic for the beauty of skin and hair, and for perfume; it complements care afforded by monoi and $p a k u$ and is essentially intended for seduction. Hoho requires a longer preparation time than paku and can take up to half a day to produce. The preparation involves numerous fresh plants which are crushed in grated coconut; no sun exposure is needed. The preparation is applied on the hair and the body without any filtration and kept for several hours or even the whole day. Hoho is not used daily but can be applied at needed. The main plants used 
for hoho are Ageratum conyzoides, Angiopteris evecta, Cocos nucifera, Curcuma longa, Mentha spp. and Ocimum basilicum.

\section{Description of particular care types}

In order to give an overview of cosmetic uses in a Marquesan life, either daily or more infrequently, several particular care types are described hereunder.

\section{Skin and hair}

Paku and monoi are mostly used for hair and body care (hydration, perfume). Daily massage is very important, providing well-being. It is also used as a cure for some illnesses. For hair care, natural shampoos are mainly made of shampoo ginger (Zingiber zerumbet), conditioner made with monoi and anti-dandruff care (paku and monoi). There are also specific preparations dedicated to promote hair growth, or to avoid hair loss. For skin problems such as eczema, injuries or fungal infections, Marquesan people used mostly tamanu oil (Calophyllum inophyllum) for three days or more if the problem persists. In the Marquesas Islands, antiperspirant preparations are often used to avoid perspiration and bad smells. Concerning acne, which is not widespread in Marquesas Islands, only a few preparations were reported. As for depilatory treatments, body hair is naturally scarce in Marquesan population, and is wildly tolerated. Only one person reported a preparation inducing hair loss, composed of leaves, fruits and stems of Leucaena leucocephala, which is well known to promote hair loss in the manes of Marquesan horses. Primarily women take care of their body and use in most cases preparations based on natural plants. In some cases, men also groom themselves by applying monoi from time to time on their body; mostly to protect themselves from twigs scratches before a hunting expedition in the bush or from the cold during fishing or navigation. Girls were trained in self-grooming and body care by their mothers, grandmothers or their aunts. For boys, after a certain age, body care is no longer considered important with the exception of frequent bathing.

\section{Baby care}

Baby care regiments are regularly followed from birth. Daily baths are given with or without boiled leaves from soursop (Annona muricata) or tamanu (Calophyllum inophyllum) for calming effect and skin care. The baby is systematically massaged, during or after the bath, in order to relax him and to ensure good development. Adding some drops of monoi in the bath provides benefits in treating skin problems as: chickenpox, prickly heat or cradle cap. Marquesan people pay attention to the healing of the navel (pito) of newborns, especially for boys: to properly dry the umbilical cord and to avoid formation of an outward navel, sandalwood powder (Santalum insulare) is applied in a compress attached to the base of umbilical cord, on the navel, for several days.

\section{Intimate hygiene}

Preparations concerning intimate hygiene are typical of the Marquesas Islands in French Polynesia. Care of private parts is very important for women starting from a very early age. These treatments are preventive actions in order to avoid bad smells and to limit vaginal discharges in adulthood. Three types of preparations were used: the first one is a paku composed of a very young green coconut or koi'e (Cocos nucifera), the second one, restricted to Ua Pou Islands, make use of roots of Achyranthes aspera, and the third one is based on a preparation using bark of tu'eiao (Rauvolfia nukuhivensis), a rare Marquesan endemic tree nearly endemic to Nuku Hiva island, and considered as an endangered species. Preparations are applied every day for several weeks, in small doses, as a few drops in the vagina, for preventive care. Later female personal hygiene consists merely of usual wash and sometimes the use of scented plants in their underwear for fragrance. These intimate care treatments are only for the female gender. Nevertheless, there is one treatment dedicated specifically for men, considered as aesthetical: the supercision.

\section{Supercision}

This traditional practice existed before the Christian missionary's arrival. In French Polynesia, and especially in the Marquesas islands, supercision or incision of foreskin (tehe in Marquesan) is a masculinity and beauty marker, and is also a hygienic practice to avoid bad odors. The equivalent treatment for female genitalia is the paku. A man who is not supercised, is usually badly considered by the community because this practice is considered a culturally significant marker of courage and virility and as a ritual passage from childhood to manhood. This practice is carried out in boys aged from 10 to 15 , by a man of the village, and never by a woman. Before the operation, genitals need to be cleaned. A hard coconut shell, or a piece of wood, was placed between the foreskin and the penis of the young boy. The skin was cut using a razor blade, or a piece of sharpened bamboo. Then, the young boy went swimming to the ocean in order to clean and heal the wound, and afterwards, he placed warm pebbles from the beach around his penis to relieve pain and to avoid inflammation. To facilitate healing, monoi is applied every day. Nowadays, circumcision takes place in hospitals and is done by nurses under medical supervision. 


\section{Tattoo}

Tattoo, tatau in Tahitian and patutiki in Marquesan, can also be considered as an aesthetic practice for both men and women of a certain age, and plants are used to make the ink mixture and to cicatrize the tattooed areas. The tattoo is not only a social status marker but also a sign of beauty and maturity. In the past, to make the ink, nuts of candlenut tree (Aleurites moluccanus) were used. Ten nuts were collected and their seeds were threaded on a rib of coconut leave. Then, seeds were sun-dried during two days and ignited in a top-down way, as a candle. A mother of pearl or an empty coconut shell was positioned above this traditional candle in order to recover fumes and soot. Soot was mixed with coconut water (Cocos nucifera), which is considered naturally sterile. Ink could also be obtained from charcoal or pounded Mucuna sloanei leaves. Tattooing is done using a wooden comb, tortoiseshells, human bones or shark teeth. Monoi may be applied after tattooing to soothe, to prevent dry skin and to help cicatrisation process.

\section{Evolution of cosmetopoeia through time}

The study of cosmetopoeia evolution was made possible only for plants biogeographical status through a literature survey of French Polynesia cosmetic plants [28]. It appears that, nowadays, more introduced plants are used, $72 \%$ versus $40 \%$ in the past, which can be considered a rapid integration of new plants and an adaptation of cosmetic uses to plants with easy access. Endemic species were more commonly used in the past with $27 \%$ of the cosmetic plants for the whole French Polynesia, in comparison with $5 \%$ of Marquesan endemics today. Apart from the high number of introduced species, the explanation is linked to the remoteness of most of these endemics which are often restricted to mountain ridges or steep slopes and which necessitate long journeys to collect them. Some of them are also becoming increasingly rare due to new threats (plants and animals invasive species) combined with some overexploitation (Rauvolfia and Alyxia bark, sandalwood). Cosmetopoeia appears to be in great evolution; with traditional practices being adapted but still thriving.

\section{Comparison with pharmacopoeia}

Marquesan cosmetopoeia and pharmacopoeia [16] have many similarities due to the lack of a clear boundary in the Marquesan traditional concepts of health and well being. The frameworks of recipes and ways of preparation are also very close and the importance of prevention is also to be noticed. The number of plant species involved is nearly identical (pharmacopoeia 77, cosmetopoeia 79) but their biogeographical status show some differences with slightly more endemic species in cosmetopoeia (4 versus 3 ) and a switch between Polynesian and modern introductions. Indeed, modern introductions are clearly preferred in cosmetopoeia (44\% versus $32 \%$ ) in comparison with Polynesian introductions, which are more numerous in pharmacopoeia (42\% versus 28\%). That indicates a higher fidelity of pharmacopoeia to traditional medicinal plants and higher innovation potential with cosmetic plants.

\section{Conclusions}

This study described the cosmetic flora of Marquesas Islands together with its uses. This field survey focusing on cosmetopoeia is the first conducted in French Polynesia, and, at the best of our knowledge, in the Pacific. Indeed, pharmacopoeia was pretty much investigated in the Pacific [29] and even in the Marquesas archipelago [16].

The current Marquesan cosmetopoeia is composed of at least 79 cosmetic plant species of which $28 \%$ are native and $72 \%$ are introduced and more or less naturalised. Most of these plant species are not threatened by overexploitation excepting three endemics: Rauvolfia nukuhivensis (bark), Alyxia stellata var. marquesensis (bark) and Santalum insulare var. marchionense - Marquesan sandalwood (heartwood). However, these species and most of the native ones face the major problem of invasive species on islands which prevent regeneration and cause the death of mature plants: rats eating seeds, herbivores grazing seedlings and eating bark, weeds and invasive plants choking juveniles and mature plants. Thus, in order to maintain traditional cosmetic uses, natural populations management of these threatened species combined with plantations to preserve the natural stock are needed. For these reasons, local authorities implemented several years ago ex situ conservation stands of Rauvolfia nukuhivensis and Santalum insulare on the island of Nuku Hiva. Our study shows that this work is highly relevant and must by pursued and amplified in the context of Marquesan cultural renewal. The main species of the Marquesan cosmetopoeia are a mixture of native species, Polynesian introductions and modern introductions, with the coconut palm (Cocos nucifera), from which the coconut oil or monoi is extracted, as the flagship species. This species assemblage indicates the innovation power of Marquesan community in front of new plants and the desire to create new cosmetic products in a traditional framework.

Fragrance appeared an important characteristic of Marquesan beauty concern. Flowers or scented materials are omnipresent in traditional cosmetic preparations (for example in monoi) or directly applied on the hair or on the skin, particularly in scented bouquet [30]. In each interview, there was at least one recipe for perfume or with deodorizing indications. Beauty also involves the softness of skin and the brightness of hair, which is 
fundamental for Polynesian women proud of their long black hair. The women, except for some specific uses (supercision, tattoo), were the main inheritors and transmitters of traditional cosmetic knowledge to following generations. Unfortunately, nowadays, knowledge transfer is partly broken, with most young Marquesans preferring ready processed products from shops or drugstores instead of preparing traditional recipes and so contributing to the preservation of Marquesan cosmetic knowledge. Thus, simultaneously with the current cultural renewal, it appeared crucial to study and collect this cosmetic knowledge, which is essentially oral.

A close link between cosmetopoeia and pharmacopoeia was demonstrated, some recipes having both cosmetic and medicinal properties. The main plants of the pharmacopoeia were also used in cosmetopoeia, like the well-known Tahitian gardenia (Gardenia taitensis).

For a better valorisation of cosmetic plants, phytochemical and specific bioassays studies could be performed. Moreover, ethnobotanical surveys regarding cosmotopoeia should be amplified in order to compare data from different archipelagos of French Polynesia and to document this important cultural wealth, both for its preservation and promotion. We attempted to meet the requirements of the Nagoya Protocol in implementing a Prior Informed Consent (PIC) form dealing also with access and benefitsharing (ABS), and in working in collaboration with Académie marquisienne, an official cultural institution of French Polynesia, aiming at preserving and enriching the Marquesan cultural heritage. Nevermind, proper regulations at the level of the French Polynesian government are needed in order to clarify the local application of access and benefitsharing concept, especially in the highly promising sector of cosmetics. Indeed, due to lack of local government regulatory framework and especially of the implementing decrees of the French Polynesian law 2012-5 from the 25 January 2012 on ABS, each study based on traditional knowledge has to develop its own ethical methods and to identify the proper representatives of traditional knowledge holders.

\section{Abbreviations}

ABS: Access and benefit-sharing; FL: Fidelity Level; NIE: Number of informants quoting that plant for any use; NIUE: Number of informants quoting one plant species for a particular use; PIC: Prior Informed Consent

\section{Acknowledgements}

We are grateful to the following:

a) Académie marquisienne - Marquesan Academy (Toti Teikiehuupoko, Julien Tamarii, Gabriel Teikitekahioho, Sarah Vaki, Léonie Peters) for their caring, for the identification of resource persons and for their help during the interviews.

b) Thomas Burelli (Ottawa University, Canada) and Tamatoa Bambridge (CNRS, CRIOBE, Moorea, French Polynesia) for the support in building the informed consent form.

c) Ranitea Ly for her help during some interviews.

d) Frédéric Jacq for the map of Marquesas Islands.

e) Soa Andrian for final English editing of the paper.

f) Cosmetic Valley for partial funding of the study.

\section{Funding}

The study was partially funded by Cosmetic Valley.

\section{Availability of data and materials}

All the available data on the cosmetic plants identified through this survey are included in the manuscript. The survey forms of each informant have been given to Académie marquisienne.

\section{Authors' contributions}

All authors designed the research network, wrote and approved the final manuscript. XJ performed interviews and wrote reports under guidance of PR, JLA and JFB. XJ built ethnobotanical datasets, statistical data and analysis under guidance of JFB, PR and GL. JFB identified plant species from botanical samples, pictures and local names.

\section{Competing interests}

The authors declare that they have no competing interests.

\section{Consent for publication}

Not applicable.

\section{Ethics approval and consent to participate}

This study was approved and supported by Académie marquisienne. Prior to each interview process, an informed consent form (Prior Informant Consent) was signed by both interviewer and informant (topic, objectives, goals of the study, respect of confidentiality).

\section{Author details}

${ }^{1}$ Ecole Nationale Supérieure Agronomique de Montpellier SupAgro, 2 pl. Viala, Montpellier Cedex 02 34060, France. ${ }^{2}$ Cosmetic Valley, 1 pl. de la Cathédrale, Chartres 28000, France. ${ }^{3}$ Ecologie Marine Tropicale des Océans Pacifique et Indien, ENTROPIE UMR 250/9220 IRD-CNRS-UR, 101, promenade Roger-Laroque, Nouméa Cedex 98848, New Caledonia. ${ }^{4}$ University of Paris Saclay - Versailles-Saint Quentin en Yvelines, 55 Avenue de Paris, Versailles 78035, France. ${ }^{5}$ University of French Polynesia, EIO UMR 241, B.P. 6570-98702 Faa'a, Tahiti, French Polynesia. ${ }^{6}$ Consultant in forestry and Polynesian botany, B.P. 52832-98716 Pirae, Tahiti, French Polynesia.

Received: 22 September 2016 Accepted: 22 November 2016 Published online: 29 November 2016

\section{References}

1. Créocéan (Coord. Seguin F) Etat de l'environnement en Polynésie française 2007/2014. Direction de l'environnement, Polynésie française. 2015. http:// www.environnement.pf/sites/default/files/diren-etat/diren-etatenvironnement-integral.pdf. Accessed 26 Nov 2016.

2. ISPF. Population légale du recensement de la population de 2012 en Polynésie française. 2013. http://www.ispf.pf/docs/default-source/publi-pr/ POP_LEGALE_2012_PF.pdf?sfvrsn=2. Accessed 6 Aug 2016.

3. Laurent V, Maamaatuaiahutapu K, Maiau J, Varney P. Atlas climatologique de la Polynésie française. Polynésie française: Météo France; 2004.

4. Butaud JF. Te Ohi o te Fenua - Guide floristique des jardins du Musée. Polynésie française: Musée de Tahiti et des lles - Te Fare Manaha; 2016.

5. Butaud JF. Hiva Oa, Tahuata, Fatuiva - Guide floristique. Polynésie française: Direction de l'environnement; 2013. http://www.environnement.pf/sites/ default/files/fichiers-documents/marquises_sud_light.pdf. Accessed 26 Nov 2016.

6. Butaud JF. Nuku Hiva, Ua Huka, Ua Pou - Guide floristique. 2nd ed. Polynésie française: Direction de l'environnement; 2013. http://www.environnement.pf/ sites/default/files/fichiers-documents/marquises_nord_0.pdf . Accessed 26 Nov 2016.

7. Wagner WL, Lorence DH. Flora of the Marquesas Islands website. 2002. http://botany.si.edu/pacificislandbiodiversity/marquesasflora/index.htm. Accessed 6 Aug 2016.

8. Ansel JL, Butaud JF, Raharivelomanana. Principaux taxons de la cosmétopée tropicale : une analyse bibliographique. C R Chimie. 2016. doi: 10.1016/j.crci. 2016.03.017.

9. Pereki H, Batawila K, Wala K, Dourma M, Akpavi S, Akpagana K, Gbeassor M, Ansel JL. Botanical assessment of forest genetic resources used in traditional cosmetic in Togo (West Africa). J Life Sci. 2012;6:931-8. 
10. Brown FBH. Flora of Southeastern Polynesia. I. Monocotyledons. Bernice P Bishop Mus Bull. 1931;84:1-194.

11. UNESCO. World Heritage Center, The List, Global Strategy, Tentative List. Les îles Marquises. 2010. http://whc.unesco.org/en/tentativelists/5564/. Accessed 6 Aug 2016.

12. Pétard P. Plantes utiles de Polynésie - Raau Tahiti. Polynésie française: Haere Po No Tahiti; 1986.

13. Whistler WA. The Ethnobotany of Tonga: The Plants, Their Tongan Names, and Their Uses. Bishop Mus Bull in Botany. 1991;2:1-155.

14. Whistler WA. Plants in Samoan culture - The ethnobotany of Samoa. Hawai'i: Isle Botanica; 2000

15. Whistler WA. Plants of the Canoe People. Hawai'i: National Tropical Botanical Garden; 2009.

16. Girardi C, Butaud JF, Ollier C, Ingert N, Weniger B, Raharivelomanana P, Moretti C. Herbal medicine in the Marquesas islands. J Ethnopharmacol. 2015;161:200-13.

17. Tardio J, Pardo-de-Santayana M. Cultural importance indices: a comparative analysis based on the useful wild plants of Southern Cantabria (Northern Spain). Econ Bot. 2008:62:24-39.

18. Friedman J, Yaniv Z, Dafni A, Palewitch D. A preliminary classification of the healing potential of medicinal plants, based on a rational analysis of an ethnopharmacological field survey among Bedouins in the Negev Desert. Israel J Ethnopharmacol. 1986;16:275-87.

19. Hoffman B, Gallahert T. Importance indices in ethnobotany. Ethnobotany Res Appl. 2007:5:201-18.

20. Charpentier JM, François A. Linguistic atlas of French Polynesia. Papeete \& Berlin: Université de la Polynésie française \& de Gruyter Mouton; 2015. http://alex.francois.free.fr/data/Charpentier-Francois_2015_Atlas-Linguistiquede-Polynesie-Francaise.pdf . Accessed 26 Nov 2016.

21. Florence J, Lorence DH. Introduction to the flora and vegetation of the Marquesas Islands. Allertonia. 1997;7:226-37.

22. Meyer JY, Butaud JF. The impact of rats on the endangered native flora of French Polynesia (Pacific Islands): drivers of plant extinction or coup de grâce species? Biol Invasions. 2009;11:1569-85.

23. Lorence DH, Butaud JF. A reassessment of Marquesan Ochrosia and Rauvolfia (Apocynaceae) with two new combinations. Phytokeys. 201 1;4:95-116.

24. UICN France, MNHN, DIREN Polynésie française. La liste rouge des espèces menacées en France - Chapitre Flore vasculaire endémique de Polynésie française. France: Paris; 2015. http://uicn.fr/wpcontent/uploads/2015/12/ Liste_rouge_Flore_vasculaire_endemique_de_Polynesie_francaise.pdf . Accessed 26 Nov 2016.

25. Butaud JF, Gaydou V, Bianchini JP, Faure R, Raharivelomanana P. Dihydroxysesquiterpenoids from Santalum insulare from French Polynesia. Nat Prod Commun. 2007:2(3):239-42.

26. Martin NJ, Prado S, Lecellier G, Thomas OP, Raharivelomanana P. Nukuhivensiums, indolo [2,3-a] quinoliziniums from the Marquesan plant Rauvolfia nukuhivensis. Molecules. 2012;17:12015-22.

27. Martin NJ, Ferreiro SF, Barbault F, Nicolas M, Lecellier G, Paetz C, Gaysinski M, Alonso E, Thomas OP, Botana LM, Raharivelomanana P. Indole alkaloids from the Marquesan plant Rauvolfia nukuhivensis and their effects on ion channels. Phytochem. 2015;109:84-95.

28. Butaud JF. Synthèse bibliographique portant sur les plantes utilisées dans la cosmétopée de Polynésie française. Study report. Tahiti: Cosmetic Valley; 2013.

29. Whistler WA. Polynesian Herbal Medicine. Hawaii: National Tropical Botanical Garden; 1992.

30. Duchek A, Kamia L, Tamarii J, Tauhiro L, Vaki S, Georges T. Te Umuhei, le bouquet odoriférant des Marquises. Tahiti: Te Pu Tuhuna Eo Enata - Editions des mers Australes; 2010

\section{Submit your next manuscript to BioMed Central and we will help you at every step:}

- We accept pre-submission inquiries

- Our selector tool helps you to find the most relevant journal

- We provide round the clock customer support

- Convenient online submission

- Thorough peer review

- Inclusion in PubMed and all major indexing services

- Maximum visibility for your research

Submit your manuscript at www.biomedcentral.com/submit 